\title{
Class prefixes as Specifiers in Southern Bantu
}

\author{
Knut Tarald Taraldsen • Lucie Taraldsen Medová • \\ David Langa
}

Received: date / Accepted: date

\begin{abstract}
We argue that a set of facts about the plural nominal class prefixes in Southern Bantu languages shows that some plural prefixes spell out a phrasal constituent, a Specifier bottoming out in a classifier-like noun. This leads us to adopt a theory of lexicalization that leads to the conclusion that all nominal class prefixes in Southern Bantu lexicalize Specifiers of this sort, and we argue that the relation between primary and secondary prefixes supports this conclusion. We also discuss the consequences of our conclusion for the pairing of prefixes and nouns and for the analysis of agreement.
\end{abstract}

Keywords Bantu nominal class prefixes · classifiers · agreement with conjoined subjects · the syntax/lexicon interface $\cdot$ Xhosa $\cdot$ Zulu $\cdot$ Changana $\cdot$ Rhonga $\cdot$ Shona

\section{Introduction}

The class prefixes that occur on nouns in Bantu languages are often analyzed as affixes added in the morphology to spell out the gender and number features associated with a noun (see in particular Bresnan and Mchombo (1995)). In this article, we wish to argue for a different view more akin to proposals by Myers (1987) and Carstens (1991) and yet also different from those proposals in important respects. While we agree that a prefix-noun combination must be seen as a syntactic construct, we take the syntactic account one step further by analyzing a class prefix not as a morpheme spelling out a single functional head, e.g. a 'little n' or Number, but rather as a morpheme lexicalizing a whole phrase introduced as the specifier of a functional head on the nominal spine. The arguments that lead us to this conclusion are based on evidence that the structures lexicalized by prefixes are projections of classifier-like nouns. As a consequence of this, we will also have a way of dealing with the often-noted fact that the class prefixes in Bantu appear to straddle the line between inflectional and derivational morphology. Our evidence comes in part from new observations regarding the relationship between the singular and the plural class prefixes in Southern Bantu. ${ }^{1}$ The following paradigm displays the nominal class prefixes in Xhosa:

Knut Tarald Taraldsen

University of Troms $\varnothing$

Tel.: +47-776-44275

E-mail: knut.taraldsen@uit.no

Lucie Taraldsen Medová

University of Troms $\varnothing$ / Troms $\varnothing$ taxi

David Langa

Universidade Eudardo Mondlane Moçambique

1 The data discussed in this article comes primarily from Xhosa, Shona and the Tsonga languages, in particular Changana. Author 1 and Author 2 have worked with 21 consultants for Xhosa (undergraduates at Stellenbosch 
(1) The paradigm for Xhosa ${ }^{2,3}$

$\begin{array}{llll}\text { Class } & \text { prefix } & \text { Class } & \text { prefix } \\ 1 & u-m- & 2 & a-b a- \\ 3 & u-m- & 4 & i-m i- \\ 5 & i(-l i-) & 6 & a-m a- \\ 7 & i-s i- & 8 & i-z i- \\ 9 & i-N- & 10 & i-z i-N / i i-N- \\ 11 & u(-l u-) & & \\ 14 & u(-b u-) & & \\ 15 & u-k u- & & \end{array}$

The initial vowels in these forms fall away in certain syntactic environments and should be analyzed as a separate morpheme often called 'the augment'. In our discussion, we will focus on the part of the complex prefix that follows the augment, and the term 'prefix' should be understood to refer exclusively to this bit.

The paradigm in (1) is organized so as to reveal a typical feature of Bantu noun class systems: Certain singular classes in the left column are paired with specific plural classes in the sense that a noun which appears as a singular in class $X$, forms the corresponding plural using the prefix of the plural class $X+1$ paired with $X$. In (1), each of the first five singular classes in the leftmost column is paired with the plural class next to it.

A simple way of capturing the singular/plural pairing is to say that the two members of a singular/plural prefix pair have the same gender features, but have different number features, as in Carstens (1991). ${ }^{4}$ The class prefixes can then be seen as different spell-outs of a number head conditioned by gender features inherited from the noun and $[-P l]$ vs. $[+P l]$. Thus, the singular/plural pairing exemplified in (1) is seen as entirely parallel to the Italian gender-based pairing in (2):

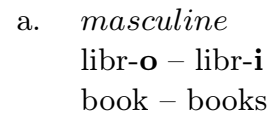
b. feminine cas-a - cas-e house - houses

Using Roman numerals to represent genders, we would have:

(3)

$\begin{array}{llll}\text { Class } & & \text { Class } & \\ 1 & u-m-=\{\mathrm{I},[-\mathrm{PL}]\} & 2 & a-b a-=\{\mathrm{I},[+\mathrm{PL}]\} \\ 3 & u-m-=\{\mathrm{II},[-\mathrm{PL}]\} & 4 & i-m i-=\{\mathrm{II},[+\mathrm{PL}]\} \\ 5 & i(-l i-)=\{\mathrm{III},[-\mathrm{PL}]\} & 6 & a-m a-=\{\mathrm{III},[+\mathrm{PL}]\} \\ 7 & i-s i-=\{\mathrm{IV},[-\mathrm{PL}]\} & 8 & i-z i-=\{\mathrm{IV},[+\mathrm{PL}]\} \\ 9 & i-N-=\{\mathrm{V},[-\mathrm{PL}]\} & 10 & i-z i-N=\{\mathrm{V},[+\mathrm{PL}]\}\end{array}$

This seems to be the dominant view in current research on Bantu, but represents a breach with the more traditional view that the connection between singular and plural classes is in general much less direct, a view partially resurrected by Schadeberg (2001) and Kihm (2005), among others. ${ }^{5}$

University and other native speakers, as explained in footnote 8), while Author 3, a native speaker of Changana, has provided the Tsonga data; the Shona data come from Fortune (1955). Unless otherwise indicated, all examples come from our field notes.

2 A class prefix preceded by an initial vowel (the 'augment'), as in (1), is omitted when the host noun is polysyllabic in classes 11 and 14 . In class 5 , the class prefix $l i$ is omitted on polysyllabic nouns even when the initial vowel is absent.

3 The $\mathrm{N}$ in classes 9 and 10 is a nasal assimilating to a following consonant, the parenthesized CV-segments fall away on polysyllabic nouns, the class 10 prefix only preserves the $z$ on monosyllabic nouns except in contexts where the initial vowel is dropped.

4 Actually, Carstens implements this by making vocabulary insertion context-sensitive, e.g. $b a \leftrightarrow\{+P l\} /{ }_{-}\{N$, gender $I\}$.

5 And Crisma et al. (2011) offers an insightful discussion of the alternatives. 
Our general conclusion regarding the nature of Bantu class prefixes will be based in part on evidence that a version of the traditional view is in fact correct only for a proper subset of the singular/plural pairs. This evidence is presented and discussed in two instalments. In section 2 , we examine agreement patterns with conjoined singular nouns, arguing that the facts show that neither class 6 nor class 4 prefixes inherit their gender features from the noun they combine with. In section 3, we introduce a formal analysis of class 4 and class 6 prefixes based on the assumption that gender features nevertheless always originate from nouns before we examine some further predictions of this analysis in section 4 and show that these predictions are borne out. In section 5, we show how the theoretical assumptions justified along the way also lead to the conclusion that all class prefixes in Southern Bantu are morphemes spelling out specifiers and argue that facts about the so-called 'secondary prefixes' provide independent support for this conclusion. In section 6 , we discuss some cross-linguistic predictions arising from our analysis.

\section{Unexpected plurals}

In analyses where all plural classes are taken to share the gender-features of the corresponding singular classes in combination with the number feature $[+P l]$, class 6 nouns are seen as the plural forms of class 5 nouns. That is, the class 6 prefix $a-m a$ - would spell out the gender-features characterizing class 5 nouns plus $[+P l]$. Here, we offer some reasons to think that this is not correct.

2.1 Not all class 6 forms are based on nouns in class 5

The fact that some nouns in class 1 and class 9 also have plurals in class 6 , suggests that the class 6 prefix $m a$ is not associated with the gender-features of class $5:^{6,7}$

$$
\begin{array}{ll}
\text { a. } & \text { u-m-Zulu } \rightarrow \text { a-ma-Zulu } \\
& \text { 1-1-Zulu 6-6-Zulu } \\
& \text { 'a Zulu' - 'Zulus' } \\
\text { b. } & \text { i-n-doda } \rightarrow \text { a-ma-doda } \\
& \text { 9-9-man 6-6-man } \\
& \text { 'a man' - 'men' }
\end{array}
$$

(Xhosa)

However, there is still a way of maintaining the claim that paired singular/plural prefixes always have the same gender-features. Adopting a proposal by Carstens (1991), Zulu might belong to a gender G such that the spell-out of $N u m=\{G,[-P l]\}$ is syncretic with the spell-out of Num $=\{I,[-P l]\}=$ $u-m$, while the spell-out of $\{G,[+P l]\}$ is syncretic with $\{I I I,[+P l]\}=a-m a-$. Likewise, doda 'man' would be assigned gender $H$, and $i$ - $N$ - spells out both $\{H,[-P l]\}$ and $\{V,[-P l]\}$, while $a-m a$ - spells out both $\{H,[+P l]\}$ and $\{I I I,[+P l]\}$. Provided that $I, I I, I I I, I V, V, G$ and $H$ are taken to represent sets of features standing in a subset relation to one another, this scheme seems easy to implement in accordance with current thinking about lexical insertion. If syncretism only moves along adjacent cells in the paradigm (see McCreight and Chvany (1991), Caha (2009), i.a.), we might have (5) (with genders renumbered):

\footnotetext{
${ }^{6}$ We use the following glosses: $\#=$ number, $\mathrm{PL}=$ plural, $\mathrm{SG}=$ singular, $\mathrm{SC}=$ subject concord, $\mathrm{M}=$ masculine, $\mathrm{F}=$ feminine, $\mathrm{N}=$ neuter, $\mathrm{DJ}=$ disjoint form. In Xhosa, the disjoint forms of the verb have the prefix $y a$ - in the present tense and the suffix -ile (active forms) or -iwe (passive forms) in the recent past tense. The disjoint forms are used when the verb is the final element in the VP; cf. Van der Spuy (1993). What the correct explanation for this may be, is orthogonal to the claims of this paper.

7 Schadeberg (2001) draws a more general conclusion from comparable facts in Swahili: Gender plays no role in the pairing of singular and plural classes. Our own conclusion in this section will be less general than this, but we will ultimately be led to adopt Schadeberg's view, see section 5.3.
} 
(5)

$\begin{array}{llll}\text { Class } & & \text { Class } & \\ 3 & u-m-=\{\mathrm{I},[-\mathrm{PL}]\} & 4 & i-m i-=\{\mathrm{I},[+\mathrm{PL}]\} \\ 1 & u-m-=\{\mathrm{II},[-\mathrm{PL}]\} & 2 & a-b a-=\{\mathrm{II},[+\mathrm{PL}]\} \\ & u-m-=\{\mathbf{G},[-\mathrm{PL}]\} & & a-m a-=\{\mathbf{G},[+\mathrm{PL}]\} \\ 5 & i(-l i-)=\{\mathrm{III},[-\mathrm{PL}]\} & 6 & a-m a-=\{\mathrm{III},[+\mathrm{PL}]\} \\ & i-N-=\{\mathbf{H},[-\mathrm{PL}]\} & & a-m a-=\{\mathbf{H},[+\mathrm{PL}]\} \\ 9 & i-N-=\{\mathrm{IV},[-\mathrm{PL}]\} & 10 & i-z i-N=\{\mathrm{IV},[+\mathrm{PL}]\} \\ 7 & i-s i-=\{\mathrm{V},[-\mathrm{PL}]\} & 8 & i-z i-=\{\mathrm{V},[+\mathrm{PL}]\}\end{array}$

If these gender-features are represented as sets of more primitive features and ordered by subset as $I \supset I I \supset G \supset I I I \supset H \supset I V \supset V$ and if lexical insertion is governed by the Superset Principle and the Elsewhere Principle (see Starke (2009) and section 3.3 below), the lexical entries in (6) will now generate (4):

$$
\begin{aligned}
& \text { a. } \quad m \leftrightarrow\{I,[-P l]\} \quad \text { b. } \quad m i \leftrightarrow\{I,[+P l]\} \\
& \text { c. } \quad b a \leftrightarrow\{I I,[+P l]\} \\
& \text { d. } \quad m a \leftrightarrow\{G,[+P l]\} \\
& \text { e. } \quad l i \leftrightarrow\{I I I,[-P l]\} \\
& \text { f. } i-N \leftrightarrow\{H,[-P l]\} \\
& \text { h. } \quad \text { si }\{V,[-P l]\} \\
& \text { g. } \quad z i \leftrightarrow\{I V,[+P l]\}
\end{aligned}
$$

(The gaps in the columns indicate the absence of a morpheme matching exactly both the number feature and the set of gender-features: In such cases, the entry immediately above the gap will be used.)

Accounting for pairs like those in (4) in terms of added genders and syncretism, is a perfectly plausible line of analysis. Outside of Bantu, this strategy appears to work well for a set of Romanian nouns which behave like masculine nouns in the singular, but like feminine nouns in the plural, e.g. deget 'finger' deget-e 'fingers'. In (7), these are taken to be neuter, but syncretize with the masculine in the singular and with the feminine in the plural:

$$
\begin{aligned}
& \text { SG PL } \\
& \text { masculine prieten-ul prieten-i-i 'the friend } / \mathrm{s} \text { ' } \\
& \text { feminine cas-a cas-e-le 'the house/s' }
\end{aligned}
$$$$
\text { neutrum deget-ul deget-e-le 'the finger/s' }
$$

But we will see in section 2.4 that the approach that works for Romanian, doesn't actually work for Southern Bantu.

\subsection{Agreement with conjoined subjects}

Our argument is based on patterns of agreement wih subjects formed by conjunction of singular nouns in the same class. ${ }^{8}$

8 The agreement data reported in this section originates from fieldwork Author 1 and Author 2 conducted with 21 native speakers of Xhosa. The fieldwork was conducted in two steps. In the first round, we consulted graduates students at Stellenbosch University (12 speakers of Xhosa: pilot study with 3 students in November 2013 and more detailed study with 9 students in May/June 2014). The next round was conducted by questionnaires that our consultant had other native speakers of Xhosa fill (April 2014). These consultants in the second round are of varying age and education and come from various socioeconomic backgrounds. Unfortunately, we were not able to keep constant one variable, namely the place of origin of the speakers. Hence, we have speakers who were born and spent their entire lives in Khayelitsha (a township of Cape Town), but also others who were born and raised in the Eastern Cape and moved to the Western Cape only later in their lives. We would need to consult way more speakers to have a clue on the dialectal variation.

Thus, for certain noun combinations, we have up to 21 speakers of Xhosa. 
To set the scene, we begin by considering a simple account of gender-sensitive agreement with conjoined singular subjects of the same gender: The gender common to the conjuncts is copied onto the agreement marker, but the number feature on the agreement marker is set to $[+P l]^{9}$. This is 'formal agreement.'

Just in case the gender of the conjoined singular nouns is in fact the same as the gender of the plural nouns they are associated with, this predicts that agreement with a conjunction of singular nouns A and B will be marked exactly the same way as agreement with the plural form of A or B. In Italian, for example, this prediction is mostly borne out (with an important exception that will come up in the next subsection):

a. I maestr-i/alunn-i sono partit-i.

the $_{M . P L}$ teachers/pupils are left- $M . P L$

'The teachers/pupils have left.'

b. Le maestr-e/alunn-e sono partit-e.

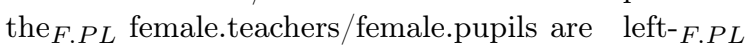

'The (female) teachers/(female) pupils have left.'

(Italian)

\footnotetext{
a. Il maestr-o e l'alunn-o sono partit-i.

the ${ }_{M . S G}$ teacher and the ${ }_{M . S G}$.pupil are left-M.PL

'The teacher and the pupil have left.'

b. La maestr-a e l'alunn-a sono partit-e.

the $_{F . S G}$ female.teacher and the ${ }_{F . S G}$.female.pupil are left- $F . P L$

'The (female) teacher and the (female) pupil have left.'
}

(Italian)

We will now investigate the consequences of assuming that this analysis is correct for subject/verb agreement in Southern Bantu.

2.3 Agreement facts sometimes support the shared gender hypothesis...

Even in Southern Bantu, the analysis suggested for Italian agreement with conjoined singular nouns sometimes gives exactly the outcome we expect, if singular nouns have the same gender as the plural nouns they are paired with. In particular, the SC ('subject concord') occurring with a subject formed by conjunction of two nouns both in class 1, 7 or 9 is identical to the $\mathrm{SC}$ chosen when the subject is a single plural noun in class 2,8 or $10:^{10}$

$$
\begin{array}{ll}
\text { a. U-m-ntwana no-m-fazi ba-ya-dlala. } \\
\text { 1-1-child and.1-1-woman SC2-DJ-play }
\end{array}
$$

For the first round of fieldwork, we conducted interviews with the consultants where the consultant was asked to fill in the correct SC in frames consisting of a conjoined subject and a verbal predicate. The second round of fieldwork complemented the first round: consultants were asked to fill in SCs in a written questionnaire and send the result back to us.

9 Equivalently, the node dominating a conjunction inherits the gender common to the conjuncts, but is always marked $[+P l]$, and the agreement marker copies the features of that node.

10 For (10-a), all of our Xhosa consultants had the SC ba (class 2). One consultant could also use the class 1 SC $u$, presumably as an instance of agreement with only one conjunct. As for (11-a), 20 out of 21 natives Xhosa speakers chose $z i$ (classes 8 and 10); two consultants also accepted ba (class 2) and one also accepted si (class 7), again, probably an instance of agreement with a single conjunct. A single consultant only accepted si. For (12-a), we obtained 16/20 zi with three consultants also accepting $i$ (class 9) and one also accepting ba (class 2). Four consultants only accepted $b a$.

From now on, information like this will mostly be conveyed by expressions of the form $x / y A(z A / B$, ...) to be read as ' $x$ consultants out of $y$ accepted $A$, and $z$ out of those $x$ consultants also accepted $B$ ', where $A$ and $B$ are $\mathrm{SCs}$ 
'The child and the woman are playing.'

b. A-ba-ntwana/a-ba-fazi ba-ya-dlala.

2-2-child/2-2-woman SC2-DJ-play

'The children / women are playing.'

(11) a. I-s-anuse ne-s-angoma zi-sebenza ndawonye.

7-7-diviner and.7-7-healer SC8-work together

'The diviner and the healer work together.'

b. I-z-anuse/i-z-angoma zi-sebenza ndawonye.

8-8-diviner/8-8-healer SC8-work together

'The diviners/healers work together.'

a. I-n-tombi ne-m-bongi zi-ya-cula.

9-9-girl and.9-9-poet SC10-DJ-sing

'The girl and the poet are singing.'

b. Ii-n-tombi/ii-m-bongi zi-ya-cula.

10-10-girl/10-10-poet SC10-DJ-sing

'The girls / poets are singing.'

(Xhosa)

This supports the hypothesis that the two members of the singular/plural pairs $1 / 2,7 / 8$ and $9 / 10$ actually do have the same gender features ${ }^{11}$ and are differentiated only by an additional number feature $([-P L]$ vs. $[+P L])$ as well as the account of agreement with conjuncts given in subsection 2.1.

\section{$2.4 \ldots$ but not always}

As mentioned in section 2.1, some class 1 nouns, e.g. umXhosa and umZulu, and some class 9 nouns, e.g. indoda 'man' and inkwenkwe 'boy', have plurals in class 6, but Carstens (1991) makes this consistent with the shared gender account of the singular/plural pairing by taking underlying gender contrasts to be neutralized at the level of spell-out. For example, umZulu and umXhosa would have a gender feature $G$ distinct from the gender feature $I I$ associated with 'real' class 1 nouns like umfazi 'woman' (paired with the class 2 plural abafazi), but $m$ spells-out both $G$ and $I I$ in the singular, while $m a$ spells out both $\{G,[+P l]\}$ and $\{I I I, P L\}$, where $I I I$ is the gender associated with class 5 nouns. $(9 / 6$ pairs like indoda/amadoda are treated in a similar manner.)

Given the way agreement with conjoined nouns was seen to work in the preceding subsection, this account of the 'irregular' class 6 plurals makes a clear prediction. An SC agreeing with umXhosa nomZulu 'a Xhosa and a Zulu' should spell out the gender feature $G$ plus the number feature $[+P L]$, and should therefore be (syncretic with) the SC6 a, exactly as with the plural subject amaXhosa in (13-b). But as $(13-\mathrm{a})$ shows, this prediction is not borne out: ${ }^{12}$
a. U-m-Xhosa no-m-Zulu ba/*a-sebenza ndawonye.
1-1-Xhosa and.1-1-Zulu SC2/SC6/work together
'A Xhosa and a Zulu work together.'
b. A-ma-Xhosa/a-maZulu a/*ba-sebenza ndawonye.
6-6-Xhosa/6-6-Zulu SC6/SC2-work together
'The Xhosas / Zulus are working together.'

\footnotetext{
11 We take the fact that $z i$ occurs both in class 8 and in class 10 to be an instance of accidental syncretism. In many other Bantu languages, e.g. the Tsonga languages discussed in section 4 , the two classes have distinct SCs and class prefixes on nouns.

12 Testing (13-a) with our Xhosa consultants gave the following outcome: 19/20 ba ( $1 \mathrm{ba} / u$ and $1 \mathrm{ba} / a$ (class 6)), $1 / 20 u$.
} 
So, it seems that umXhosa and umZulu must have the gender-features associated with classes 1 and 2 rather than any set of gender features that can be spelled out by the class 6 prefix $m a$ and the SC6 $a-$ even though the plurals paired with umXhosa and umZulu actually have ma.

Nor can the gender-features of indoda 'man' and inkwenke 'boy' be a subset of those associated with $m a$ and $a$ in spite of the fact that they are paired with the class 6 plurals amadoda, amakwenkwe: ${ }^{13}$

a. I-n-doda ne-n-kwene zi/*a-ya-cula. 9-9-man and.9-9-boy SC10/SC6-DJ-sing

'A man and a boy are singing.'

b. A-ma-doda/a-ma-khwenkwe a-ya-cula. 6-6-man/6-6-boy SC6-DJ-sing

'The men / boys are singing.'

(Xhosa)

Instead, the gender-features of indoda and inkwenkwe seem to be those of the class $8 / 10$ prefix $z i$ rather than those of the class 6 prefix ma.

Thus, if the singular prefixes in (13)-(14) inherit their gender-features from the noun, the plural prefix $m a$ cannot always have the same gender-features as the noun it combines with.

The argument leading to this conclusion is identical to the argument used by Acquaviva (2008) to show that Italian singular/plural pairs like il dito/le dita 'finger', il braccio/le braccia 'arm', l'uovo/le uova 'egg' etc. do not belong to a third gender which happens to have the same exponents as the masculine in the singular, but the same exponents as the feminine in the plural. Based on the agreement algorithm justified by (8)-(9), that assumption incorrectly predicts that the participle should show feminine plural inflection in (15-a) exactly as in (15-b):

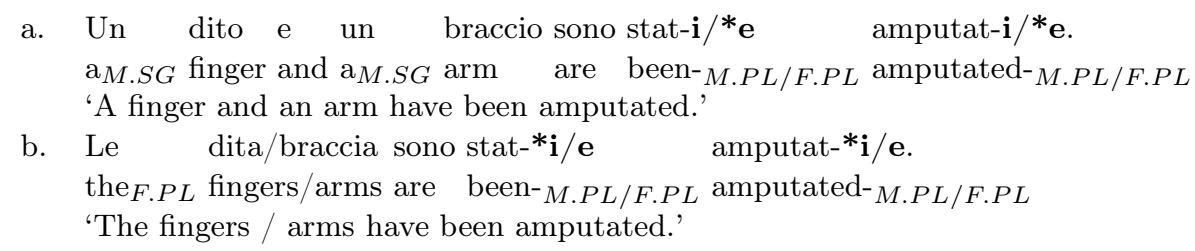

(Italian)

Therefore, the singular noun is simply a masculine noun while the plural noun it is paired with, is feminine. That is, the singular and the plural form do not have the same gender in pairs like il dito/le dita. $^{14}$

\footnotetext{
13 The outcome for (14-a) was: 14/19 zi (2 zi/ba, $2 z i / i), 3 / 19 b a, 2 / 19 i$. tian Bican for confirming the judgment).

(i) a. Un deget și un brațau fost amputat-*i/e.

$\mathrm{a}_{M . S G}$ finger and $\mathrm{a}_{M . S G}$ arm have been amputated-* ${ }_{M . P L} / F . P L$

'A finger and and arm have been amputated.'

b. Degetele / brațele au fost amputat-*i/e.

fingers-the $_{F . P L} /$ arms-the $_{F . P L}$ have been amputated-* ${ }_{M . P L} / F . P L$

'Fingers and arms have been amputated.'
}

14 By the same token, (i-a) supports, as Acquaviva points out, the contention that Romanian pairs like deget/degete 'finger', brat,/brațe 'arm' really do belong to a third gender ('neuter') in Romanian (thanks to Sebas- 
2.5 No singular class has the same gender-features as the class 6 prefix

We have argued that the class 6 prefix $m a$ doesn't have the same gender-features as umXhosa, umZulu or indoda, inkwenkwe in spite of the fact that the regular plurals of these nouns have the class 6 prefix $m a$. We will now show that class 6 forms cannot have the same gender-features as class 5 nouns either, although class 6 is the plural class canonically paired with class 5. The crucial fact is that the SC6 $a$ never appears with a subject formed by conjunction of class 5 nouns: ${ }^{15}$

a. I-li-tye ne-qanda $\mathbf{z i} / * \mathbf{a}$-khataza i-n-taka. 5-5-stone and.5-egg SC8/SC6-annoy 9-9-bird 'The stone and the egg annoy the bird.'

b. A-ma-tye a-khataza i-n-taka. 6-6-stone SC6-annoy 9-9-bird 'The stones annoy the bird.'

(Xhosa)
a. I-gqirha ne-gosa ba/*a-sebenza ndawonye.
5-healer and.5-steward SC2-work together
'The healer and the steward are working together.'
b. A-ma-gqirha a-sebenza ndawonye.
6-6-healer SC6-work together
'The healers are working together.'

(Xhosa)

Instead, the SC chosen is predominantly $\mathrm{SC} 2 b a$ when one of the conjoined nouns denotes human beings, but otherwise SC9/10 zi. ${ }^{16}$ This is in fact the same pattern we see with conjunctions of singular nouns belonging to different classes, ${ }^{17}$ i.e. when formal agreement as characterized in section 2.2 necessarily fails. In that case, the gender-features of the conjuncts cannot be copied onto the SC which therefore is selected by a different criterion (reference to humans). But in (16)-(17), where both conjuncts belong to class 5 , there should be no problem with copying the gender-features of the conjuncts onto the SC. What leads to the animacy-based selection of the SC in this case, must be that there simply is no spell-out available for a $\mathrm{SC}$ associated with the gender-features of class 5 plus the number feature $[+P l]$. That is, there happens to be no morpheme associated with that particular combination of features. In particular, SC6 $a$ cannot be associated with a feature set containing the gender-features of class 5 in addition to $[+P l]$, and since $a$ always occurs under agreement with the class 6 prefix $m a,{ }^{18}$ we conclude that $m a$ isn't associated with those gender-features either.

15 Only two of 21 Xhosa consultants accepted SC6 $a$ in (16-a) and (17-a), in both cases as a possible second (third) option, never as a 'first choice'. According to Corbett and Mtenje (1987), a conjunction of two class 5 nouns doesn't trigger agreement in class 6 in Chichewa either. As in Xhosa, default agreement appears instead.

16 For (16-a), where both nouns are inanimate, the outcome was: 15/20 zi (2 zi/li and $1 z i / a$ (class 6)), 5/20 li (1 li/a (class 6$)$ ). For (17-a), with two nouns denoting human beings, the outcome was: 10/20 $b a(2 b a / z i$ and 1 $b a / l i), 7 / 20 z i(1 z i / b a$ and $1 z i / l i), 3 / 20 l i(1 \mathrm{li} / z i)$.

17 With a conjunction of singular nouns from different classes where both nouns denote human beings, all 12 Xhosa speakers consulted chose SC2 $b a$ for $1+3,1+5,1+7,3+5,7+1,7+5,9+1$. (One consultant could also have a singular SC agreeing with the first conjunct.) With $3+5$ where both nouns were inanimate, all consultants preferred $\mathrm{SC} 8 / 10 z i$.

Agreement with a conjunction of nouns from different singular classes in Bantu languages has been studied by Givón (1970), Corbett and Mtenje (1987), Marten (2000, 2005), i.a. Unlike Voeltz (1971), we did not find that speakers of Xhosa reject subjects formed by nouns of different classes. Instead, our informants used SC2 and SC8 for default agreement essentially as in Kuria as described in Diercks et al. (2015) (except that Diercks et al. don't classify this use of $\mathrm{SC} 2$ as a default strategy).

18 Only 5 out of 20 Xhosa speakers failed to get SC6 $a$ for $6+6$, for combinations of animate+animate and inanimate+inanimate and animate+inanimate, the alternative form was always $b a$ (regardless of the combination of animate and inanimate nouns in the input). A similar pattern was identified for Chichewa by Givón (1970) and confirmed by Corbett and Mtenje (1987). 
Thus, it appears that no singular prefix has the same gender features as the class 6 prefix. Hence, if the singular prefixes inherit the gender of the noun, the class 6 prefix does not.

\subsection{Class 4}

An analogous conclusion can be reached for class 4 . Class 4 is normally taken to be the plural class corresponding to class 3 . In fact, only class 3 nouns have plurals in class 4 . So, it seems eminently plausible that the class 4 prefix is associated with PL plus the gender-features of class 3 .

Yet the expected SC4 $i$ never occurs when the subject is a conjunction of two class 3 nouns. Instead, one mostly gets $\mathrm{SC} 2 b a$ when the subject denotes human beings, and SC8 $z i$ otherwise, just as with conjoined class 5 nouns: ${ }^{19}$

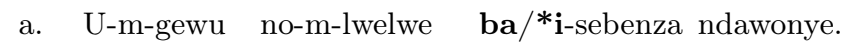
3-3-criminal and.3-3-cripple SC2/SC4-work together 'A criminal and a cripple are working together.'

b. I-mi-gewu i-sebenza ndawonye. 4-4-criminal SC4-work together.

'The criminals are working together.'

a. U-m-bhingqo no-m-nqathe $\mathbf{z i} / * \mathbf{i}$-se-tafile-ni.

(Xhosa) 3-3-skirt and.3-3-carrot SC8/SC4-table-LOC 'A skirt and a carrot are on the table.'

b. I-mi-bhinqo i-se-tafile-ni. 4-4-skirt SC4-table-LOC 'The skirts are on the table.'

(Xhosa)

In other words, it seems that the gender-features of class 4 are not in fact the same as the gender-features of class 3 , in spite of appearances to the contrary. In section 4, we will present another type of evidence confirming this conclusion.

\section{$2.7 b a$ and $z i$ are not always defaults}

Before we conclude this section, we need to exclude an alternative analysis of the agreement patterns attributed to formal agreement in section 2.3. We have seen that SC2 $b a$ and SC8 zi also occur as default SCs when formal agreement must fail. Then, $b a$ is used when one of the conjoined nouns denotes human beings, and $z i$ is used elsewhere. Since most class 1 nouns denote persons, this suggests that the use of SC2 $b a$ with a conjunction of class 1 nouns as in (10-a) might also be the default $b a$, selected on the basis of semantic compatibility rather than by formal agreement, contrary to what we assumed in section 2.3. Likewise, the use of SC8 $z i$ with conjoined singular nouns in class 7 or 9 might be based merely on semantic compatibility to the extent that class 7 nouns and class 9 nouns do not denote persons. That is, it might be that formal agreement always fails with conjoined subjects in Xhosa in which case the facts discussed in section 3 tell us nothing about the gender of $m a$ and $m i$.

However, some class 1 nouns, e.g. $u L$ 'the letter L' and other names for the letters of the alphabet, do not seem to denote persons, but still a conjunction of two such nouns also requires SC2 ba in Xhosa, ${ }^{20}$

\footnotetext{
19 (18-a) outcome: 11/11 ba (1 ba/u (class 3)), (19-a) outcome: 13/21zi (2 zi/u and 1 zi/a (class 6)), 5/21 u (3 $u / z i$ and $1 u / i$ (class 4)), 3/21 ba. Again, Corbett and Mtenje (1987) claim that Chichewa is similar.

20 For an example translating ' $\mathrm{L}+\mathrm{M}$ are in the picture', 18 out of 20 speakers had $b a(3 b a / u$ and $1 b a / z i)$. Class 6 marker $a$ was suggested by one speaker and so was a class 8 marker $z i$ (3 others suggested $u$ as a second option, i.e. $3 b a / u$, as indicated above).
} 
and conversely, some nouns in class 7 and 9 do denote persons, but a conjunction of them still requires SC8 $z i$, as shown by examples like (11-a) and (12-a).

As for the fact that even a conjunction of two inanimate class 1 nouns comes with SC2 ba, one might say that such nouns are 'personified'. However, one can disprove this conjecture for Xhosa by looking at what happens when the subject is a conjunction of an (apparently) inanimate class 1 noun and an inanimate noun from a different class. This is the scenario in which the use of default $b a$ or $z i$ is called for, and it turns out that both $b a$ and $z i$ are possible in Xhosa when one of the conjoined nouns denotes persons, and the other doesn't - but not when both are inanimate. ${ }^{21}$ Our data indicates that the ratio of $b a$ 's with a conjunction of an inanimate class 1 noun and an inanimate noun of a different class is lower than with a conjunction of an animate class 1 noun and an inanimate noun of another class. ${ }^{22}$ The fact that $b a$ occurs at all, of course suggests that some speakers really do personify $u L$ etc., but - crucially - some speakers don't, given the lower number of $b a$ 's compared to the situation where the class 1 noun clearly refers to persons, and yet all our Xhosa consultants have SC2 $b a$ with a conjunction of two inanimate nouns both in class 1 . Taken together with the fact that Xhosa speakers uniformly also have $z i$ even with conjunctions of two person-denoting nouns in class 7 or 9 , this supports the view that the choice of the SC is really determined by formal agreement in the Xhosa sentences discussed in section 2.2 .

\subsection{Summary}

The main conclusion reached in this section is that the distribution of SCs agreeing with conjunctions of singular nouns is consistent with adopting the shared gender hypothesis for the singular/plural pairs $1 / 2,7 / 8$ and $9 / 10$, but is inconsistent with adopting this hypothesis for pairs involving class 6 or class 4 .

Since the same root occurs in both the singular and the plural member of pairs like umZulu 'Zulu' / amaZulu (1/6), indoda 'man' / amadoda (9/6) and ilitye 'stone' / amatye (5/6) or umgewu 'criminal' / imigewu $(3 / 4)$, this entails that the gender-features associated with the prefix cannot be inherited from the root in both members of the pair. Since the agreement facts examined here suggest that classes 1 and 2 have the same gender-features, and similarly for classes 7 and 8 as well as 9 and 10, it is eminently plausible that the gender-features of the prefix are in fact inherited from the root in these cases. Thus, we end up with the conclusion that the gender-features of the class 4 prefix $m i$ and the class 6 prefix $m a$ aren't inherited from the root. This leads to the following question:

(20) What is the source of the gender-features associated with the class 6 prefix $m a$ and the class 4 prefix $m i$ ?

In the next section, we develop an answer to this question.

\section{Binominal structures and silent nouns}

We have argued that the gender features associated with the pluralizing prefixes of class 4 and 6 are not the same as those associated with the corresponding singular classes 3 and 5 - or any other singular class. We will now show that - first - this contradicts standard claims about the source of gender features unless

21 Thus, Xhosa differs from Sotho as described by De Vos and Mitchley (2012), where conjunction of a noun denoting persons with an inanimate noun cannot appear as a preverbal subject.

${ }^{22}$ For instance, 10 out of 10 consultants have $\mathrm{SC} 2 b a$ when an inanimate class 9 noun is conjoined with an animate class 1 noun, but only 3 out of 11 have $b a$ when an inanimate class 9 noun is conjoined with an inanimate class 1 noun. (The $b a$ might pick up gender from a single conjunct in this case.) This seems parallel to the fact that a conjunction of an inanimate class 3 noun with an inanimate class 5 noun comes with the SC 8/10 zi for 10 out of 11 consultants, while a conjunction of an animate class 3 noun and an inanimate class 5 noun gives SC2 $b a$ for 8 out of the same 11 consultants. 
nouns in class 4 and 6 have binominal structures, and then develop a specific account of the properties of those structures incorporating the basic ideas of Nanosyntax (see Starke (2009) for a general outline).

3.1 The gender features of class 4 nouns and class 6 nouns don't come from the host noun

We begin by highlighting the consequences of our findings for current accounts of how gender-features are assigned to class prefixes in Bantu.

As already mentioned in section 2.1, Carstens (1991) introduced a simple structural account of how gender features come to be associated with the heads that are spelled out by class prefixes on nouns. The prefix $m$ of class 1 nouns like $u$ - $m$-fazi 'woman', for example, would spell out a number head (notated as \# in (21) and further) associated with the number feature and a gender feature $I$ inherited from the nominal root fazi: ${ }^{23}$

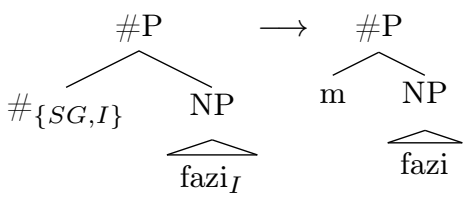

The class 2 prefix $b a$, on the other hand, spells out \# associated with $\{P L, I\}$, as shown in (22).

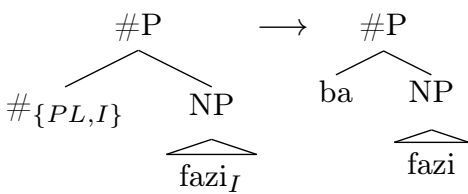

That is, gender is inherent to nouns and spreads to prefixes by agreement.

Similarly, the class 5 prefix $l i$ in (23) would spell out \# with the number feature SG and the gender feature $I I I$ inherited from the noun $f u$ 'cloud':

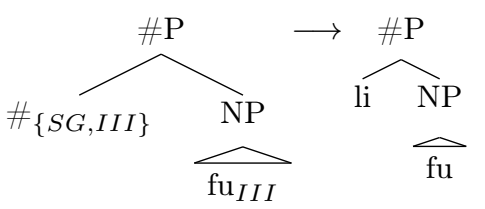

But then, what about class 6 plurals like $a$-ma-fu 'clouds'? Since the noun is the same as in the corresponding class 5 singular $i$-li-fu 'cloud', amafu should be associated with the same gender feature $I I I$ as ilifu:

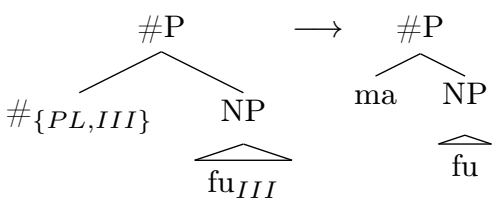

But the agreement facts discussed in section 2.4 show that class 6 forms do not have the same gender as any singular class, and likewise for the class 4 forms, excluding also the pairing in (25) for $u$ - $m$-thi (class 3) 'tree' and $i$-mi-thi 'trees':

\footnotetext{
23 We will ignore the augment $u$, whose form is determined by the basic prefix.
} 


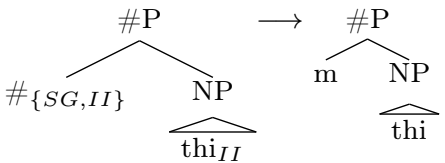

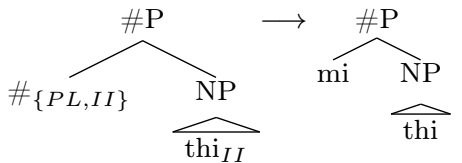

That is, we are forced to conclude that plural prefixes in class 4 and class 6 do not inherit the gender features of the nouns they seem to combine with. ${ }^{24}$

Yet, the prefixes $m i$ and $m a$ must be differentiated in terms of gender, if they both have the same number feature PL. So, where do their gender features come from? This is the question first formulated in (20). What we have seen now is just that we cannot sidestep this question simply by assuming that $m i$ and $m a$ are not associated with gender-features.

\subsection{Binominal structures}

If we want to maintain the widely shared assumption that a gender feature always originates from a noun, the only possible answer to this question is that the structures underlying class 4 forms and class 6 forms have two nouns in them, e.g. as in (26) or (27), where the number head \# inherits the gender feature of $\mathrm{N}_{X}:^{25}$

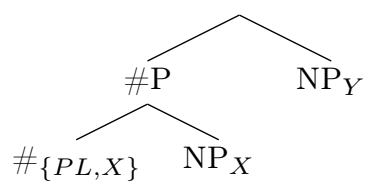

$\mathrm{N}_{X}$ itself will not be pronounced (separately from the prefix). In the next subsection, we offer an explanation why this is so.

Then, $m a$ and $m i$ have different gender if they combine with silent Ns with different genders (arbitrarily named 4 and 6):

(27)

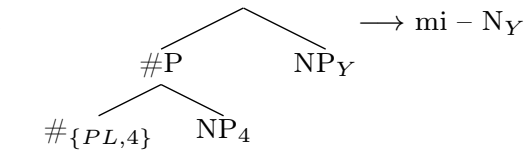

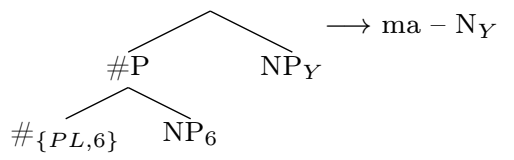

This is the core of our answer to the question in (20) repeated in (28):

(28) What is the source of the gender-features associated with the class 6 prefix $m a$ and the class 4 prefix mi?

24 On the basis of similar facts concerning the singular/plural pairing in Swahili, Schadeberg (2001) draws an analogous conclusion. Outside of Bantu, Kihm (2005) and Lecarme (2002) make the same point for Manjaku and Somali. In the words of Lecarme (2002:119): "What is crucial here is that in the plural we are dealing with gender values which are not from the noun. This gender value must therefore be a feature of the plural suffix itself." (Kihm and Lecarme's proposals are discussed in section 6.)

25 The alternative structure in (i) would be incompatible with the theory of lexicalization adopted below (subsection 3.4):

(i)

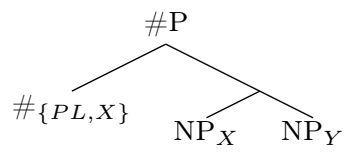

We will want the prefix to lexicalize the two heads \# and $N_{X}$, but on our theory of lexicalization lexical insertion can only target constituents. 
In (26)-(27), the silent $\mathrm{N}$ determining the gender associated with the class prefix is inside a Specifier of the overt $\mathrm{N}$. The alternative representation in (29), where the overt $\mathrm{N}$ is the complement of the silent $\mathrm{N}$, would make the incorrect prediction that the linker $a$ 'of' should appear in front the overt $\mathrm{N}$ as in other structures where an N-projection if the complement of an N, e.g. (30). ${ }^{26}$

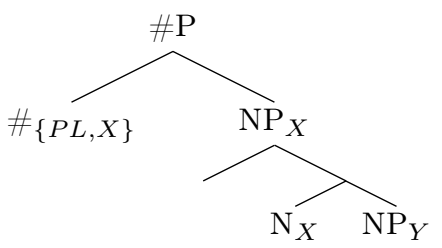

(30) i-qela l-a-ma-Xhosa

5-group SC5-of.6-6-Xhosa

'a group of Xhosas'

Also, the structure in (29) would be inconsistent with the upcoming explanation (in the next subsection) for why $\mathrm{N}_{X}$ is silent.

\subsection{Phrasal lexicalization}

We would now like to propose that $\mathrm{N}_{X}$ in (26), repeated here as (31), is not really silent, but is spelled out by the same morpheme that lexicalizes $\#_{\{P L, X\}}$ :

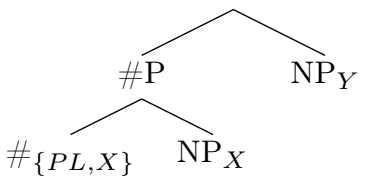

That is, we assume that a single morpheme can lexicalize more than a single terminal. In (32), the entire Specifier is lexicalized by $m i$ in $(32-\mathrm{a})$ and by $m a$ in $(32-\mathrm{b}):^{27}$
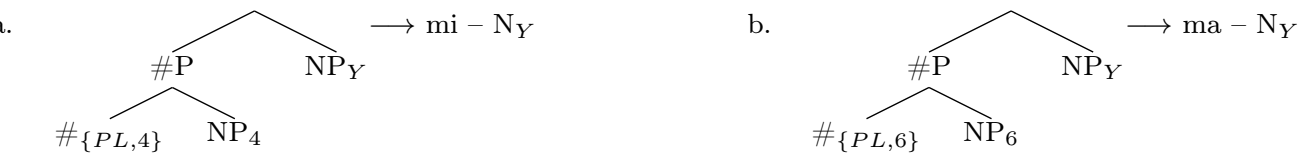

An obvious way of allowing a single morpheme to spell out a non-trivial set of terminals is to allow lexical insertion to target phrasal nodes, as discussed by Starke (2009), Caha (2009) and Pantcheva (2011). When a morpheme M replaces two terminals X and Y, lexical insertion applies to the node labeled XP in (33) and the lexical entry for M will be as in (34):<smiles>[Y][Y7][Y]</smiles>

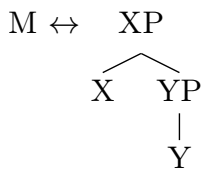

Lexical insertion is licensed by a matching relation between a structure built by the syntax and the structure associated with a morpheme in the lexicon:

(35) Matching

A syntactic structure $S$ matches a morpheme $M$ with the entry $M \leftrightarrow S^{\prime}$ if and only if

\footnotetext{
26 The surface form of the linker $a$ 'of' is affected by a phonological process coalescing $a$ 'of' with a following augment: / a a / $\rightarrow[\mathrm{a}], / \mathrm{a} \mathrm{i} / \rightarrow[\mathrm{e}], / \mathrm{a} \mathrm{u} / \rightarrow[\mathrm{o}]$. The class 6 augment is $a$.

27 This possibility was suggested to us by Michal Starke (personal communication) as an alternative to the analysis in Taraldsen (2010).
} 
the root node $R$ of $S$ has the same label as some node $N$ in $S^{\prime}$ and

(i) every daughter of $R$ matches a daughter node of $N$, and

(ii) every daughter of $N$ matches a daughter node of $R$

Notice that according to (35), the $M$ with the entry in (34) can also lexicalize just YP. Thus, the so-called Superset Principle in (36) is a direct consequence of Matching rather than an independent principle:

\section{The Superset Principle}

A morpheme $M$ associated in the lexicon with a syntactic structure $\Sigma$ can lexicalize any syntactic structure matching a constituent of $\Sigma$.

Notice also that it follows from (35) that two adjacent terminals XY can only be lexicalized by M when they form a constituent. ${ }^{28}$ Therefore, a single morpheme with the entry in (37) can spell out \# $\{P L, X\}$ and $\mathrm{N}_{X}$ together in (31), but not in (38):
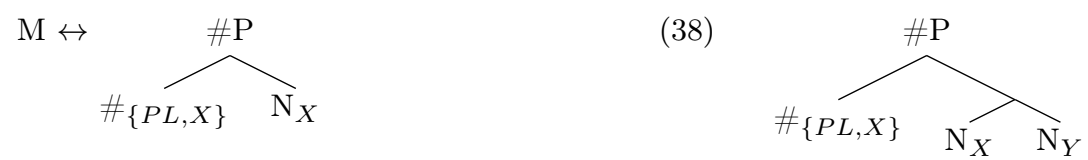

The reason is that the phrasal node immediately dominating $\mathrm{N}_{X}$ in (38) has a daughter $\mathrm{N}_{Y}$ which finds no match in the structure associated with $\mathrm{M}$ in (37).

Matching, as stated in (35), does not, however, exclude using the M in (37) to spell out the two heads $\#_{\{P L, X\}}$ and $\mathrm{N}_{X}$ separately in accordance with the Superset Principle targeting only terminal nodes. This would yield $\mathrm{MMN}_{Y}$ both from (31) and (38). In the relevant literature, e.g. Abels and Muriungi (2008), this possibility is excluded by an independent principle constraining the Superset Principle: ${ }^{29}$

\section{The Foot Condition}

If $\mathrm{M}$ has the lexical entry $\mathrm{M} \leftrightarrow[\mathrm{X}[\ldots[\mathrm{Z}]]]$, any piece of syntactic structure lexicalized by $\mathrm{M}$ must contain $\mathrm{Z}$.

Therefore, if the lexicon contains the entry in (37), $\mathrm{M}$ can only lexicalize $\#_{\{P L, X\}}$ as part of a constituent containing $\mathrm{N}_{X}$. Therefore, $\#_{\{P L, X\}}$ and $\mathrm{N}_{X}$ must be lexicalized as a unit by a single $\mathrm{M}$ in (31), and $\mathrm{M}$ cannot lexicalize $\#_{\{P L, X\}}$ in (38).

This has a further consequence which we note here, although it will not be crucial to what follows. Consider again the representations in (32) repeated below as (40):

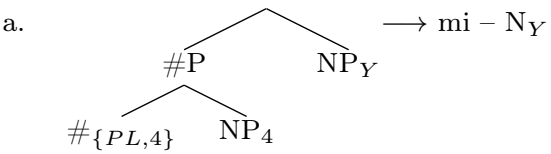

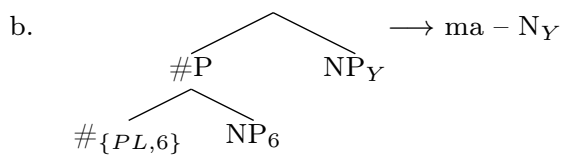

Here, the number head \# is associated with different gender features represented by numbers. But this is just an unnecessary vestige of Carsten's (1991) analysis where the prefix is taken to spell out just \# so that the choice between different morphemes lexicalizing \# need to be conditioned by a gender feature associated with \#. We have argued that in classes 4 and 6 , this gender feature must come from a silent $\mathrm{N}_{4}$ or $\mathrm{N}_{6}$, as in (40). In this section, we have also argued that silent $\mathrm{N}_{4}$ and $\mathrm{N}_{6}$ must be lexicalized

\footnotetext{
28 In contrast to this, 'spanning' would also allow a single morpheme to spell out a set of terminals that do not form a constituent. 'Spanning' can be traced back to McCawley (1968). In its current form, its closest ancestor is Williams (2003). See Abels and Muriungi (2008), Taraldsen (2010) and Svenonius (2012b) for some recent applications. Reasons for not choosing this alternative will appear at various points in the text.

29 The effect of (39) for right-branching trees would follow from Matching as defined in (35) only if insertion of a morpheme associated with a phrase (rather than a single terminal) in its lexical entry could only target phrasal nodes.
} 
together with \# by a single morpheme, and this makes it unnecessary to associate a gender feature with \#. Given the lexical entries in (41), the choice between $m i$ and $m a$ is conditioned simply by the difference between $\mathrm{N}_{4}$ and $\mathrm{N}_{6}$ :

$$
\text { a. } \quad m i \leftrightarrow \overbrace{\#\{P L\}}^{\# \mathrm{P}} \overbrace{\mathrm{N}_{4}}
$$

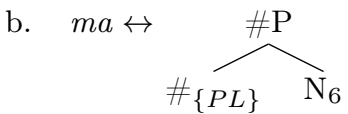

Thus, we will take it that no gender feature is associated with \# in the structures lexicalized by $m i$ and $m a$. Actually, as far as classes 4 and 6 are concerned, gender may play no role at all in determining the spell-out, since the two Ns in (41) presumably cannot belong to different genders without there being other differences between them. If so, the subscripted numbers on $\mathrm{N}$ in (41) should not be regarded as representing gender features, but merely as encoding a distinction between two different Ns.

\subsection{Features and portmanteau morphemes}

Putting aside the specific issues concerning classes 4 and 6, we now turn to the analysis of the plural classes that are paired with singular classes in a predictable way, e.g. class 2 with the prefix $b a$. The plurals in this class always correspond to singular nouns in class 1. As we have already seen, Carsten (1991) captures this correspondence by saying that $b a$ lexicalizes $\#\{P L\}$ associated with the same gender feature as the class 1 prefix $m$, i.e. $\#_{\{P L, I\}}$ :

$$
\text { a. } m \leftrightarrow \#\{S G, I\} \quad \text { b. } \quad b a \leftrightarrow \#_{\{P L, I\}}
$$

On the assumption that the gender feature originates from the $\mathrm{N}$ hosting the prefix, this ensures that all nouns combining with $b a$ also combine with $m$.

However, bundling a number feature and a gender feature on \# runs counter to proposals by Kayne (2005), Kayne (2016), Starke (to appear), who argue that each head should associate with only one feature. A system merging features one by one as syntactic heads seems simpler than a system that precompiles features into bundles subsequently used as atomic building blocks in syntactic structures.

From this perspective, a number feature \# and a gender feature $\mathrm{G}$ can only co-occur in structures like (43), where the two features correspond to two different heads:

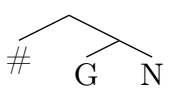

This raises an issue about the nature of portmanteau morphemes that express both gender and number like the Bantu class prefixes, e.g. the $m$ and $b a$ in (42). Theories that maintain a one-to-one correspondence between morphemes and syntactic heads, e.g. Distributed Morphology, typically appeal to Fusion, an operation turning two adjacent heads into one before Vocabulary Insertion, but this comes with the problem that Fusion must somehow be constrained so as to apply only when there is a morpheme matching its output, as observed by Chung (2009) and others. This complication is avoided in theories that allow a single morpheme to spell out more than one head, but the specific theory of phrasal lexicalization described in subsection 3.3 would not actually allow a morpheme $\mathrm{M}$ to lexicalize both \# and $\mathrm{G}$ to the exclusion of $\mathrm{N}$ in (43), where \# and G don't form a constituent.

It would, however, allow it in (44) derived from (43) by raising the N (provided this movement doesn't leave a trace visible to the lexicalization procedure; cf. Pantcheva (2011) and Starke (2009, to appear)):

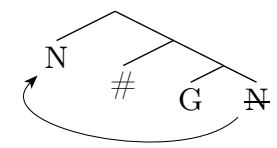


But an $\mathrm{M}$ replacing \# and $\mathrm{G}$ in (43) will be a suffix, and the Bantu class prefixes are portmanteau prefixes. It seems that the only way of having \# and $\mathrm{G}$ lexicalized by single morphemes such as the class $1 \mathrm{~m}$ and the class $2 b a$ is by assuming that \# and $\mathrm{G}$ are grouped together as a Specifier of N:<smiles>[1H]CC([CH])CN</smiles>

But (45) is inconsistent with the generally accepted assumption that functional heads are only merged on top of a lexical head. In particular, nominal functional heads like \# and G should only be merged on top of an N. Therefore, (45) should be replaced by (46):

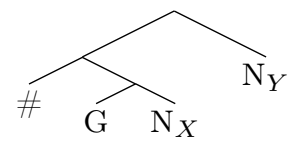

That is, regularly paired plural class prefixes should also be taken to lexicalize a Specifier containing a 'silent' $\mathrm{N}$ just like class 4 and class 6 prefixes. If so, the gender feature $\mathrm{G}$ becomes redundant as pointed out in the preceding subsection, because there must be a distinct $\mathrm{N}_{X}$ for each plural class. Thus, (46) reduces to $(47)$ :

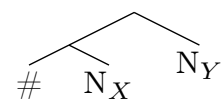

To capture the fact that all plural forms with the class 2 prefix $b a$ correspond to singular forms with the class 1 prefix $m$, we must then assume that $b a$ and $m$ lexicalize Specifiers containing the same $\mathrm{N}_{X}$, i.e. if \# is privative PL, we'll have:

$$
\text { a. } \quad m \leftrightarrow\left[\mathrm{N}_{1}\right]
$$

b. $\quad b a \leftrightarrow \widehat{\# \mathrm{~N}_{1}}$

Similarly for classes 7 and 8 :
a. $\quad s i \leftrightarrow\left[\mathrm{N}_{7}\right]$
b. $\quad z i \leftrightarrow \widehat{\mathrm{N}}_{7}$

But the $\mathrm{N}_{X}$ inside the Specifiers lexicalized by the class 4 prefix $m i$ and the class 6 prefix $m a$ will not also appear in any Specifiers spelled out by singular prefixes, e.g. we'll have the entries in (48) for the class 5 prefix $l i$ and the class 6 ma:
a. $\quad l i \leftrightarrow\left[\mathrm{N}_{5}\right]$
b. $\quad m a \leftrightarrow \widehat{\mathrm{N}}_{6}$

As shown in the following subsection, this accounts for the agreement facts discussed in section 2.

While we based our analysis of the class 4 prefix $m i$ and the class 6 prefix $m a$ on the empirical facts examined in section 2, the extension of this analysis to all class prefixes suggested here, is so far motivated only by adopting two theoretical proposals: A non-trivial set of heads can be jointly lexicalized by a single morpheme just in case they form a constituent (as in subsection 3.3) and each syntactic head corresponds to a single feature. But empirical motivation for the extension will appear in section 5 based on the observation that the regular class prefix of some class is often also used as a so-called 'secondary prefix' with nouns of a different class acting like diminutive or augmentative affixes. This fact prompted Carstens $(1991,2008)$ to posit a silent $\mathrm{N}$ when a prefix is used as a secondary prefix. We will argue that the facts discussed in section 5 are best captured by positing the same silent $\mathrm{N}$ also when the prefix is used as a regular class prefix. 
$3.5 \mathrm{SCs}$

If the nominal class prefixes are gender-less, as suggested above, what we called formal agreement in section 2.2, must involve copying the $\mathrm{N}_{X}$ inside the class prefix into the $\mathrm{SC}$ rather than copying gender features. The fact that a verb always has the $\mathrm{SC} 2 b a$ when its subject is a conjunction of two class 1 nouns, and always has the SC8 $z i$ when the subject is conjunction of two class 7 nouns, is then captured by having formal agreement copying the $\mathrm{N}_{X}$ of $m$ (class 1) and si (class 7), i.e. $\mathrm{N}_{1}$ and $\mathrm{N}_{7}$, into the structure spelled out by the SC and adding the pluralizing \#. SC2 ba and SC8 $z i$ will then be morphemes with exactly the same lexical entries as the corresponding nominal class morphemes (the examples are repeated from (48) and (49) above):

$$
\begin{array}{ll}
\text { a. } & m \leftrightarrow\left[\mathrm{N}_{1}\right] \\
\text { b. } \quad b a \leftrightarrow \widehat{\# \mathrm{~N}_{1}}
\end{array}
$$

$$
\begin{array}{ll}
\text { a. } & \text { si } \leftrightarrow\left[\mathrm{N}_{7}\right] \\
\text { b. } \quad z i \leftrightarrow \widehat{\# \mathrm{~N}_{7}}
\end{array}
$$

The fact that formal agreement fails when the subject is a conjunction of class 3 nouns or class 5 nouns follows from confining the $\mathrm{N}_{X}$ inside the class 3 and class 5 prefixes, i.e. $\mathrm{N}_{3}$ and $\mathrm{N}_{5}$ to lexical entries where they do not combine with \#. For example, $\mathrm{N}_{5}$ in (53-a) does not also occur in (53-b):

$$
\text { a. } \quad l i \leftrightarrow\left[\mathrm{N}_{5}\right] \quad \text { b. } \quad m a \leftrightarrow{\widehat{\# \mathrm{~N}_{6}}}_{\mathrm{N}_{6}} \quad \text { c. } \quad a \leftrightarrow \overparen{\# \mathrm{~N}_{6}}
$$

Therefore, copying $\mathrm{N}_{5}$ from a conjunction of class 5 nouns into the SC adding \# will result in a structure that cannot be spelled out. In particular, it cannot be spelled out by SC6 $a$ which we take to have an entry similar to the nominal class 6 prefix $m a$ in (53-b), i.e. (53-c) and therefore only occurs with a subject in class $6 .^{30}$

But the conclusion that SCs too contain a 'silent' $\mathrm{N}_{X}$ just like the nominal class prefixes actually follows at least in part from the analysis of nominal prefixes adopted in the preceding subsection quite independently of whether or not the nominal class prefixes are gender-less (include a $\mathrm{G}$ encoding a gender feature). If all the nominal prefixes lexicalize some $\mathrm{N}_{X}$ (the singular prefixes) or [\# [ $\left.\left.\mathrm{N}_{X}\right]\right]$ (the plural prefixes), regardless of whether the structures they lexicalize, also contain a $G$, the theory of lexicalization adopted in subsection 3.3 forces us to conclude that SCs too lexicalize some $\mathrm{N}_{X}$ or [\# [ $\mathrm{N}_{X}$ ]]. This is because many SCs are identical to class prefixes on nouns. For example, SC2 ba and SC8 $z i$ are identical to the class prefixes on nouns in class 2 and class 8 , respectively. The simplest explanation for this is that the same morpheme actually does double duty as a SC and a class prefix on nouns as we already assumed at the beginning of this subsection. But even if the nominal prefixes $b a$ and $z i$ associated with the entries in (54) (with the gender feature G retained), $b a$ and $z i$ will be unable to lexicalize [ \# [ $\mathrm{G}_{X}$ l] (with $\mathrm{X}=1$ or 7 ) in (55) given the Foot Condition:

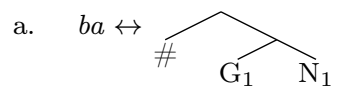

b.<smiles>[18CH]CC([Al])C[Al]</smiles>

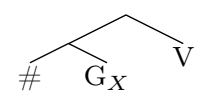

The Foot Condition

If $\mathrm{M}$ has the lexical entry $\mathrm{M} \leftrightarrow[\mathrm{X}[\ldots[\mathrm{Z}] \mid]$, any piece of syntactic structure lexicalized by $\mathrm{M}$ must contain $\mathrm{Z}$.

\footnotetext{
30 Given that $a$ and $m a$ have the same structure, we might ask why aren't they interchangeable. The best answer is probably that $m a$ is bimorphemic and properly contains the SC $a$. That is, the prefix on nouns would have the structure [ $\left.\mathrm{X}\left[\#\left[\mathrm{~N}_{6}\right]\right]\right]$ with $\mathrm{X}$ lexicalized by $m$ and [ \# [ $\left.\left.\mathrm{N}_{6}\right]\right]$ lexicalized by $a$. The $\mathrm{X}$ would be lexicalized by $m$ in all the weak classes, i.e. classes $1,3,4$ and 6 , but there is problem with classes 1 and 3: the prefix should be $m u=\left[\mathrm{X}(=m)\left[\mathrm{N}_{1,3}(=u)\right]\right]$ (though SC1 and $\left.3=u\right)$. The prefixes of strong classes, e.g. class $2 b a$, would then be morphemes that can lexicalize all of $\left[\mathrm{X}\left[\#\left[\mathrm{~N}_{X}\right]\right]\right]$ or $\left[\mathrm{X}\left[\mathrm{N}_{X}\right]\right]$.
} 
Moreover, [ \# [ $\left.\left.\mathrm{G}_{X}\right]\right]$ in (55) would contain two nominal functional heads not merged on top of an $\mathrm{N}$ just like the previously discussed (45) (repeated below):

$$
\overbrace{\mathrm{G}} \mathrm{N}
$$

Therefore, the structures underlying SCs $b a$ and $z i$ must also contain $\mathrm{N}_{1}$ and $\mathrm{N}_{7}$ respectively:<smiles>CCC1CCCC1C#N</smiles>

b.

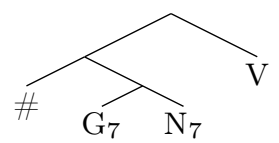

Formal agreement must therefore involve copying the $\mathrm{N}_{X}$ of the prefix of class 2 and class 6 nouns controlling agreement, and, consequently, the gender head $\mathrm{G}$ becomes redundant for the spell-out of the SCs $b a$ and $z i$ as well. (58), then, reduces to (59) with the entries for $b a$ and $z i$ repeated from (48)-(49) (and replacing (54)):

a.<smiles>[1H]C([1H])(C[3H])C[3H]</smiles>
a. $\quad m \leftrightarrow\left[\mathrm{N}_{1}\right]$
b. $\quad b a \leftrightarrow \widehat{\# \mathrm{~N}_{1}}$

b.<smiles>[3H]CC([3H])([3H])C[3H]</smiles>

$$
\begin{array}{ll}
\text { a. } & \text { si } \leftrightarrow\left[\mathrm{N}_{7}\right] \\
\text { b. } & z i \leftrightarrow \widehat{\mathrm{N}}_{7}
\end{array}
$$

Whether this second line of reasoning also leads to the conclusion that all SCs contain a $\mathrm{N}_{X}$, depends on what the correct analysis of the SCs in the 'weak classes' turns out to be (we offer a glimpse of a direction in footnote 30).

We return to the SCs in section 5.4 in connection with our discussion of secondary prefixes.

\subsection{Summary}

On the basis of the agreement facts discussed in section 2, we have argued that the class 4 prefix and the class 6 prefix must correspond to a set of heads including a silent $\mathrm{N}$. In order to explain why this $\mathrm{N}$ is silent, we have also adopted a specific theory of phrasal lexicalization that forced us to assume that the heads lexicalized by the class 4 prefix or the class 6 prefix must form a constituent merged to the host noun as a Specifier. Combining our theory of lexicalization with the assumption that each syntactic head corresponds to a single feature then led us to the conclusion that all plural prefixes lexicalize Specifiers containing an $\mathrm{N}$ distinct from the host noun. The fact that gender features become redundant then suggests that singular prefixes also lexicalize an $\mathrm{N}$.

We will regard the silent Ns lexicalized by the class prefixes as classifier-like and return to this idea in section 5.3 after we have provided more evidence for the syntactic analysis proposed in this section.

\section{Stacking}

In a number of Bantu languages, a class prefix may stack on top of another class prefix. In this section, we first examine data from the Tsonga languages Rhonga and Changana in which a plural prefix stacks on top of a singular prefix in exactly those classes where formal agreement with a conjunction of singular nouns fails in the way described for Xhosa in sections $2.5-2.6$. Then, we show how this correlation is captured within the theory of lexicalization adopted in section 3.3. Finally, we examine a case where a 
plural prefix seems to stack on top of another plural prefix in Shona and argue that our analysis handles this instance of stacking too. ${ }^{31}$

\subsection{Stacking in Tsonga languages}

In the Tsonga languages Rhonga and Changana, some plural prefixes stack on top of the singular prefix in classes 3, 5 and 9. The following examples from Rhonga come from Bachetti (2006):

$$
\begin{array}{lll}
\text { a. } & \text { rhi-tu } & \text { ma-rhi-tu } \\
& \text { 5-word } & \text { 6-5-word } \\
& \text { 'word' } & \text { 'words' } \\
\text { b. } & \text { mu-twa } \longrightarrow & \text { mi-mu-twa } \\
& \text { 3-thorn } & \text { 4-3-thorn } \\
& \text { 'thorn' } & \text { 'thorns' } \\
\text { c. } & \text { yi-n-dlu } & \text { ti-yi-n-dlu } \\
& \text { 9-9-house } & \text { 10-9-9-house } \\
& \text { 'house' } & \text { 'houses' }
\end{array}
$$

(Rhonga)

Similar examples are found in Changana.

In general, the cases where stacking does not occur overtly in these classes, should probably be characterized in phonological terms. For example, we see the following pattern in Changana: ${ }^{32}$

$$
\begin{array}{lll}
\text { a. } & \text { ri-gaga } \longrightarrow \text { ma- } \varnothing \text {-gaga } & \text { 'green fruit' } \\
\text { b. } & \text { ri-gava } \longrightarrow \text { ma- } \varnothing \text {-gava } & \text { 'calf' } \\
\text { c. } & \text { ri-bze } \longrightarrow \text { ma-ri-bze } & \text { 'stone' } \\
\text { d. } & \text { ri-to } \longrightarrow \text { ma-ri-to } & \text { 'word' }
\end{array}
$$

(Changana)

In general, it seems that the class 5 prefix is retained after ma only when the root is monosyllabic. This is clearly a phonological effect reminiscent of the fact that the class 10 prefix $z i$ drops out after the initial vowel (the augment) in Xhosa, when the root is polysyllabic, but reappears in contexts where the initial vowel is dropped. Although we don't fully understand how this alternation arises, the fact that it is contingent upon the number of syllables in the root demonstrates its phonological nature. It should be emphasized that the $r i$ in (63-b) is clearly not just an 'epenthetic prefix' inserted to comply with a 'minimal word requirement' demanding that the noun have at least two moras, ${ }^{33}$ since $r i$ is never inserted with monosyllabic nouns not in class 5 , and $m a$ would supply the second mora all by itself. There is therefore no reason to assume that the $r i$ in $(63-b)$ is any other than the $r i$ in (63-a), and the correct interpretation of the facts is that this $r i$ must be elided when it follows $m a$ and the noun is

\footnotetext{
31 As a reviewer makes us aware, there is a kind of stacking in Souther Bantu as well, as described by Buell (2009) and Halpert (2015). There is a noun class transposition in Zulu, where a root+prefix combination can receive an additional noun class prefix, as shown by examples below. (i) is from Buell; it is rather similar to Manyika form vu-mu-nhu (u-bu-ntu in Xhosa) 'humanity' mentioned in section 5.1. (ii) is from Halpert: proper names are often formed by retaining the prefix from a noun and adding the class 1a augment. Such examples are also found in Xhosa. However, these cases are clearly of a different type from those discussed in this section.
}

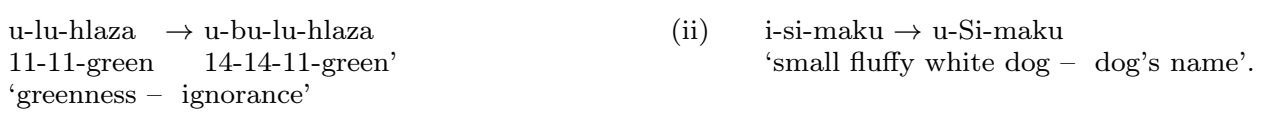

32 The Changana examples are provided by Author 3 .

33 This suggestion was made by an anonymous reviewer. 
polysyllabic (just like $z i$ in class 10 in Xhosa must be elided when preceded by an augment and followed by a polysyllabic stem).

Otherwise, the spell-out of the class 5 prefix embedded under $m a$ is determined by the same phonological properties that determine its spell-out when there is no $m a$ on top, e.g. it always spells out as $\varnothing$ on polysyllabic stems with an initial continuant or sonorant:

$$
\varnothing \text {-sema } \longrightarrow \text { ma-Ø-sema 'smell' }
$$

(Changana)

For our purposes, the most interesting fact about stacking in Changana and Rhonga is that only occurs in class 4,6 and 10. Although the class 1 prefix $m u$ has the same phonological shape as the class 3 prefix, the class 2 prefix $v a$ (or any other plural prefix) cannot be added on top of $m u$. For example, $m u-n h u$ 'person' is paired with $v a-n h u$ 'people' rather than *va-mu-nhu. Likewise, the class 8 prefix swi is never added on top of the class 7 prefix $x i$ : Xi-fambu 'shoe' is paired with swi-fambu 'shoes', not *swi-xi-fambu.

The prefixes that allow stacking have another property not shared by the prefixes that don't stack. As in Xhosa, a conjunction of two class 3 nouns doesn't trigger class 4 agreement, and a conjunction of two class 5 nouns doesn't trigger class 6 agreement. Rhonga and Changana differ from Xhosa in that a conjunction of class 9 nouns doesn't trigger class 10 agreement either, ${ }^{34}$ but, as in Xhosa, a conjunction of class 1 noun triggers class 2 agreement, and a conjunction of class 7 nouns comes with agreement in class 8 . So, stacking occurs exactly when the singular class prefix isn't paired with a plural prefix in a regular way. We will now show how this correlation is captured within the theoretical framework described in section 3.3 .

\subsection{Derivations}

To set the scene, we begin by introducing Starke's (to appear) idea that the lexicalization procedure interacts with the process of building syntactic structures in specific ways. To illustrate, we follow the process of assembling a Bantu noun step by step.

Given a noun $\mathrm{N}_{Y}$, the first thing that happens is that $\mathrm{N}_{Y}$ merges with a classifier-like $\mathrm{N}_{X}$ :

$$
\mathrm{N}_{X} \mathrm{~N}_{Y}
$$

This simply reflects the fact that a Bantu noun needs a class prefix, and that a singular class prefix is just a morpheme lexicalizing some $\mathrm{N}_{X}$ in our analysis. We adopt Starke's assumption that an attempt is made to lexicalize a head as soon as it has been merged. ${ }^{35}$ So, if $N_{Y}$ is $n h u$ 'person' (in Changana and Rhonga) and $\mathrm{X}=1$, we get $m u$-nhu.

The derivation of a plural noun also involves merging the head \# $(=\mathrm{PL}) .{ }^{36}$ One option would be to merge \# on the $\mathrm{N}_{Y \text {-spine: }}$

$$
\overbrace{\mathrm{N}_{X}} \overbrace{\mathrm{N}_{Y}}
$$

But suppose that all morphemes that can lexicalize \# have lexical entries including some $\mathrm{N}_{X}$, as in (67).

34 This difference between the Tsonga languages and Xhosa might be related to the fact that the class 10 prefix is identical to the class 8 prefix in Xhosa, but not in Rhonga and Changana.

35 Technically, lexicalization at this stage of the derivation consists in associating syntactic structures with pointers to matching morphemes which will only replace the lexicalized structures at a later stage. Hence, a lexicalized head may remain accessible to syntactic operations.

${ }^{36}$ We take this order of Merge $\left(\mathrm{N}_{X}\right.$ before \#) to be determined by a functional hierarchy in which \# is higher than classifiers. 


$$
M \leftrightarrow \widehat{\# \mathrm{~N}_{X}}
$$

Since $\#$ and $\mathrm{N}_{X}$ don't form a constituent in (66), $\mathrm{M}$ cannot lexicalize $\#$ together with $\mathrm{N}_{X}$, and because of the Foot Condition, it also cannot lexicalize \# in isolation from $\mathrm{N}_{X}$ :

(68) The Foot Condition

If $\mathrm{M}$ has the lexical entry $\mathrm{M} \leftrightarrow[\mathrm{X}[\ldots[\mathrm{Z}]]]$, any piece of syntactic structure lexicalized by $\mathrm{M}$ must contain $\mathrm{Z}$.

Thus, \# cannot be lexicalized in (66).

Lexicalizing \# together with $\mathrm{N}_{X}$ would be possible, if $\mathrm{N}_{Y}$ raised to a position above \# giving (69), but since class markers are not suffixes in Bantu, we assume that this option cannot be taken: ${ }^{37}$

$(69)$<smiles>[Y19]C([Y])C[18N]</smiles>

Therefore, the derivation now 'backtracks' from (66) putting \# and $\mathrm{N}_{X}$ back in the work space where they are merged to form [ \# [ $\left.\left.\mathrm{N}_{X}\right]\right]$, a phrase which is then merged with $\mathrm{N}_{Y}$ :

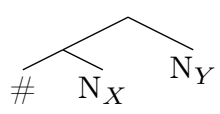

Like Starke (to appear), we take the merger of a Specifier headed by \# to extend the $\mathrm{N}_{Y}$-projection in the same way as adding just the head \# to the $\mathrm{N}_{Y}$-spine as in (66). We also follow Starke in assuming that 'backtracking' of the sort just described only applies when needed to provide a structure that can be fully lexicalized

In (70), \# is lexicalized by the $\mathrm{M}$ in (67) together with $\mathrm{N}_{X}$, as required by the Foot Condition. For example, if $\mathrm{X}=1$, it is lexicalized together with $\mathrm{N}_{X}$ by the class 2 prefix $v a$ as in $v a-n h u$ 'persons' in Changana. 38

But suppose that the classifier-like $\mathrm{N}$ merged at the first step of the derivation is some $\mathrm{N}_{Z}$ that only occurs in the entry in (71) so that M' only occurs in singular forms:

$$
M^{\prime} \leftrightarrow\left[\mathrm{N}_{Z}\right]
$$

Then, \# cannot be lexicalized even in a structure similar to (70):

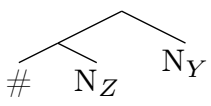

But \# can be lexicalized, if we leave $\#$ and $\mathrm{N}_{Z}$ in the work space till we have built another Specifier inside the first one, provided $\mathrm{N}_{W}$ in (73) occurs in the entry in (74):

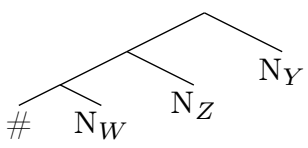

$$
\mathrm{M} " \leftrightarrow \widehat{\mathrm{N}}_{W}
$$

\footnotetext{
37 As before, we proceed on the assumption that traces are not visible to the lexicalization procedure as in Pantcheva (2011) and Starke (2009, to appear). As for why Bantu disallows the movement leading to (69), we remain agnostic, but see the suggestions in Starke (to appear: 351-354).

38 More precisely, since each head is lexicalized (in the sense of footnote 35 ) as soon as it is introduced, $\mathrm{N}_{1}$ is associated with a pointer to the class 1 prefix $m u$ before \# is merged to form the Specifier in (70). Then, since the result of merging \# creates a structure matching the class 2 prefix va, a pointer to va is associated with the root node of the Specifier, and the pointer previously associated with $\mathrm{N}_{1}$ is erased.
} 
Then, M" will lexicalize [ $\left.\#\left[\mathrm{~N}_{W}\right]\right]$ and $\mathrm{M}$ ' will lexicalize $\mathrm{N}_{Z}$ producing a stack of two prefixes on $\mathrm{N}_{Y}$ :

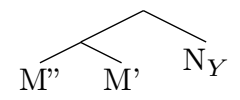

Since backtracking (of the sort described above) is taken to apply only when needed to create structures that can be fully lexicalized, the step of the derivation replacing (72) with (73) can only be taken when no morpheme has a lexical entry combining $\mathrm{N}_{Z}$ with the plural head \#. The classifier-like Ns with this property can be detected by looking at agreement facts like those discussed in section 2: If the SC cooccurring with a subject formed by conjunction of two singular nouns in the same class $\mathrm{Z}$ cannot be the same SC that occurs with the corresponding plural nouns, the classifier $\mathrm{N}_{Z}$ inside those singular nouns cannot be the same classifier that occurs in the corresponding plural forms. That is, there can be no lexical entry like (74) with $\mathrm{N}_{Z}$ instead of $\mathrm{N}_{W}$. In this case, backtracking from (72) to (73) is needed to provide a structure that can be lexicalized.

Conversely, if a subject formed from two conjoined singular nouns in some class X co-occurs with the same SC as the corresponding plural nouns, those plural nouns must use the same classifier-like $\mathrm{N}_{X}$ as the corresponding plural nouns. and therefore, there must be a morpheme with an entry like (74) with $\mathrm{N}_{X}$ instead of $\mathrm{N}_{W}$, and this morpheme can lexicalize the structure in (72) (with $\mathrm{N}_{Z}=\mathrm{N}_{X}$ ) directly. Since backtracking leading from (72) to (73) can only apply when needed to achieve full lexicalization, it won't apply in this case.

Since stacking of a plural prefix on top of a singular prefix is taken to reflect backtracking leading from (72) to (73), it now follows that a plural prefix in class Q stacks on a singular prefix in class X only if a subject conjoining two singular nouns in class X doesn't co-occur with the plural class Q SC.

The agreement facts show that the class 3 prefix $m u$, the class 5 prefix ri/rhi and the class 9 prefix $y i$ in Changana and Rhonga must have lexical entries like M' in (71), since a subject formed by conjunction of two class 3 nouns, two class 5 nouns or two class 9 nouns doesn't occur with the same SC as the corresponding plural forms (in class 4,6 and 10):

$$
\begin{array}{ll}
\text { a. } & m u \rightarrow\left[\mathrm{N}_{3}\right] \\
\text { b. } & r i / r h i \rightarrow\left[\mathrm{N}_{5}\right] \\
\text { c. } & y i \rightarrow\left[\mathrm{N}_{9}\right]
\end{array}
$$

The pluralizing prefixes $m i$ (class 4), $m a$ (class 6) and $t i$ (class 10) must then have the entries in (77) with $\mathrm{N}_{4}, \mathrm{~N}_{6}$ and $\mathrm{N}_{10}$ distinct from the $\mathrm{Ns}$ in (76):
a. $\quad m u \rightarrow \widehat{\mathrm{N}}_{4}$
b. $\quad m a \rightarrow \widehat{\mathrm{N}}_{6}$
c. $\quad t i \rightarrow \widehat{\mathrm{N}}_{10}$

Hence, the cases of stacking described above in subsection 4.1 receive a straightforward analysis similar to $(75)$ within the theory of lexicalization we adopt: ${ }^{39}$

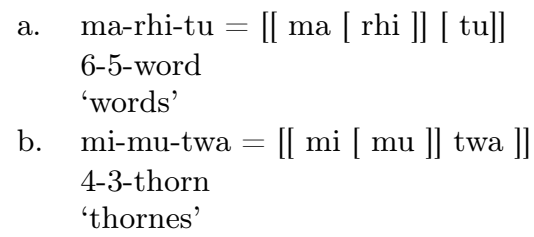
c. $\quad$ ti-yi-n-dlu $=[[$ ti [ yi $]]$ n-dlu $]]$ 10-9-9-house 'houses'

(Rhonga)

\footnotetext{
39 We put aside that status of the homorganic nasal, here - $n$-, that always immediately precedes the noun in classes 9 and 10, since this issue seems to have no bearing on the point made in the text. As for the reasons why Rhonga and Changana allow the stacking patterns 4-3- and 6-5-, but not *6-3- or *4-5-, see the discussion in section 5.3.
} 
We can also account for the fact that stacking does not occur in classes 2 and 8 . As already pointed out, class 2 is regularly paired with class 1 and class 8 is regularly paired with class 7 , and this is captured in our analysis by having the same $\mathrm{N}_{1}$ occurring both in the structure lexicalized by the class 2 prefix and in the one corresponding to the class 1 prefix as well as the same $\mathrm{N}_{7}$ both in the structure lexicalized by the class 8 prefix and in the structure lexicalized by the class 7 prefix:
a. $\quad m u \leftrightarrow\left[\mathrm{N}_{1}\right]$
a. $\quad x i \leftrightarrow\left[\mathrm{N}_{7}\right]$
b. $\quad v a \leftrightarrow \widehat{\# \mathrm{~N}_{1}}$
b. $\quad$ swi $\leftrightarrow \widehat{\mathrm{N}}_{7}$

Therefore, once the derivation has built the Specifiers in (81), \# can be lexicalized together with the following $\mathrm{N}_{X}$ :

$$
\text { a. } \widehat{\mathrm{N}}_{1} \rightarrow(\operatorname{class} 2)
$$

b. $\widehat{\mathrm{N}}_{7} \rightarrow($ class 8$)$

Accordingly, lexicalization of \# does not require building another Specifier as it did in the classes where stacking occurs, and we cannot account for the impossibility of stacking in classes 2 and 8 by taking the introduction of Specifiers not motivated by purely syntactic requirements to be a last resort option as proposed by Starke (to appear).

Finally, notice that the derivation leads to stacking in classes 4,8 and 10 only because $\mathrm{N}_{3}, \mathrm{~N}_{5}$ or $\mathrm{N}_{9}$ are merged at the initial step. But this is not the only option. In particular, $\mathrm{N}_{4}, \mathrm{~N}_{6}$ or $\mathrm{N}_{10}$ could have been merged instead:

a. $\widehat{\mathrm{N}_{4}} \mathrm{~N}_{Y}$

Then, when \# is introduced forcing backtracking and the creation of a Specifier, we would get structures where \# can be lexicalized as in classes 2 and 8 without the formation of another Specifier:

$$
\text { a. } \overbrace{\# \mathrm{~N}_{4}}^{\text {class } 4} \rightarrow m i
$$

b. $\quad$ class 6

$$
\overbrace{\# \mathrm{~N}_{6}} \rightarrow m a
$$

c. $\mathrm{N}_{10} \mathrm{~N}_{Y}$

Thus, there would be no stacking. Since Changana and Rhonga in fact require stacking, we must therefore assume that the option of introducing $\mathrm{N}_{4}, \mathrm{~N}_{6}$ or $\mathrm{N}_{10}$ at the first step in the derivation of a plural form cannot be taken in these languages. But at the same time, we can account for the fact that Xhosa disallows stacking even in classes 4 and $6^{40}$ by allowing Xhosa to take this option in preference of initiating a derivation leading to stacking. Why the Tsonga languages and Xhosa differ in this way, has to remain an open question for now, but this has no impact on our account for why the Tsonga languages show stacking exactly in the classes they do.

\subsection{Double plurals}

As described by Fortune (1955: 78), Shona allows the prefix $m a$ to attach on top of $m i$ to form a 'plural of plurals: ${ }^{41}$

40 Stacking would in any event be unexpected in class 10 in Xhosa, since a conjunction of class 9 nouns allow formal agreement in this language.

41 Another example (drawn from fieldwork by Author 3 on the Manyika dialect of Shona spoken in Moçambique) is in (i):

(i) $\begin{aligned} & \text { ma-mi-ti } \\ & \text { 6-4-tree } \\ & \text { 'groves' }\end{aligned}$ 


$$
\begin{aligned}
& \text { ma-mi-sha } \\
& \text { 6-4-village } \\
& \text { 'groups of villages' }
\end{aligned}
$$

Within the analysis of stacking in Changana and Rhonga in the preceding subsection, examples like (84) seem problematic. The first step of the derivation would merge sha with $\mathrm{N}_{4}$ yielding the structure in (85):

$$
\mathrm{N}_{4} \mathrm{~N}_{Y}
$$

Then, the introduction of the pluralizing \# would lead to backtracking and the creation of a Specifier to be merged with $\mathrm{N}_{Y}$ :

$$
\widehat{\mathrm{N}}_{4}
$$

But at this point, we find ourselves in the scenario described at the end of the last subsection, if $m i$ has the lexical entry in (87) in Shona:

$$
m i \leftrightarrow \widehat{\mathrm{N}}_{4}
$$

Since $m i$ would then be able to lexicalize \# together with $\mathrm{N}_{4}$ in (86), the last resort option of forming a Specifier could not be taken again, and (88) should be underivable:

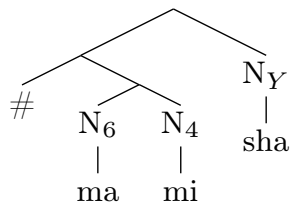

(88) is derivable, however, if $m i$ has the entry in (89) in Shona rather than the one in (87):

$$
m i \leftrightarrow\left[\mathrm{N}_{4}\right]
$$

Then, $m i$ cannot in fact lexicalize \# in Shona, and the introduction of the second Specifier in (88) is motivated.

Positing (89) instead of (87) also entails that the interpretation of class 4 nouns like $m i$-sha 'villages' in Shona cannot come from a \#. Rather, it must come from the lexical meaning of $\mathrm{N}_{4}$ itself, and therefore $\mathrm{N}_{4}$ must mean something like 'plurality'. The meaning of (84) seems straightforwardly consistent with this: $\mathrm{N}_{4}$ in (88) denotes pluralities and [ \# [ $\mathrm{N}_{6}$ ]] forms pluralities of pluralities.

In support of this analysis, we note that if the plural reading 'villages' came from a \# lexicalized by $m i$ in (88), the plural reading 'groups' would presumably come from a second \# lexicalized by ma unless $\mathrm{N}_{6}$ rather $\mathrm{N}_{4}$ is analyzed as a 'lexical plural'. But if \# occurs twice, there must be two independent extended N-projections as in (90), where the extended projection of sha 'village' is the complement of a silent lexical $\mathrm{N}_{Z}$ initiating the higher extended N-projection :

(90)

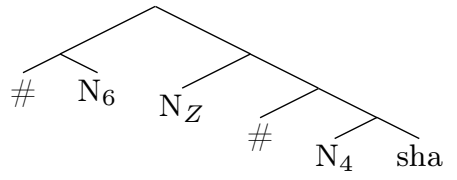

(90), however, would be exactly like the structure of (91), which requires the linker $a$ 'of': 
(91) i-qela l-a-ma-Xhosa 5-group SC5-of.6-6-Xhosa

'group of Xhosas',

(Xhosa)

If $\mathrm{N}_{6}$ is a lexical plural and does not lexicalize \# in (88), while $m i$ does, the same problem arises, if a classifier-like $\mathrm{N}$ must be introduced before \# within a single extended N-projection, as assumed above. In addition, both ways of fitting in a \# lexicalized by $m i$ in (84) open up for the possibility that there might be cases of stacking similar to (84), but with a singular class prefix on top of $m i$. Such structures would simply relate to (84) the way a group of villages relates to groups of villages, but Fortune provides no examples of this sort, and that is consistent with the analysis pursued here: Stacking, reflecting the insertion of a second Specifier, is only licensed by the need to lexicalize \#.

For the same reason, positing (89) as the lexical entry for $m i$ in Shona also predicts that there will be no forms with $m i$ stacking on top of $m a$, i.e. $m i-m a-N$, and, indeed, Fortune gives no examples of this sort. $^{42}$

4.4 Déchaine et al. (2014)

The analysis of Shona nominal class prefixes in Déchaine et al. (2014) bears a certain resemblance to the analysis proposed here. In particular, the functional heads on top of the noun are divided into two structural layers, and prefixes can occur in both layers: ${ }^{43}$

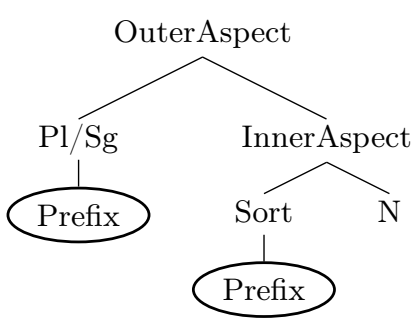

The number head $\mathrm{Pl} / \mathrm{Sg}$ is in the higher layer just like our \#, while Sort is in the lower layer like our $N_{X}$. Sort is taken to select entities in the denotation of $\mathrm{N}$ with properties determined by the specific prefix associated with it, a property we will associate with the $N_{X}$ level in section 5.3. Unlike us, however, Dèchaine et al. treat prefixes as heads rather than specifiers, and we will return to this difference shortly.

Clearly, Dèchaine et al.'s account also predicts the possibility for prefixes to stack with one prefix instantiating Sort and the other $\mathrm{Pl} / \mathrm{Sg}$, and they show that this prediction is borne out in Shona by class 14 nouns that form plurals by adding the class 6 prefix ma on top of the class 15 prefix $u$. They do not also discuss instances of stacking where the second prefix is in class 3 or 5 (as in the Tsonga cases analyzed in the subsections 4.1-4.2), but in fact their analysis seems to predict this possibility, too. Crucially, they assume that prefixes in these classes can associate both with $\mathrm{Pl} / \mathrm{Sg}$ and with Sort:

\footnotetext{
42 Given the fact that mi stacks on top of the class 5 prefix $r i / r h i$ in Changana and Rhonga, the entry for $m i$ in these Tsonga languages must still be like (87) rather than (89). That is, we assume that the pluralizing effect of $m i$ in Changana and Rhonga comes from \#, while it comes from the semantic of $\mathrm{N}_{4}$ itself in Shona.

43 They also posit a third 'evaluative' layer for some of the secondary prefixes we discuss in the next section.
} 
(93)

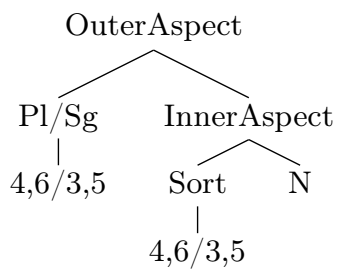

Thus, the Changana and Rhonga examples discussed in section 4.1 could be analyzed as in (94), and the Shona example in section 4.3 could have the structure in (95):

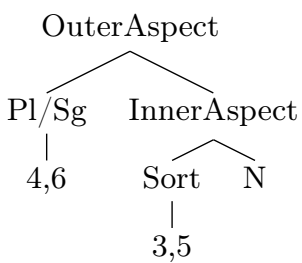

$(95)$

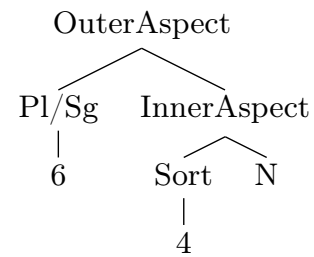

However, a difference between the two proposals emerges when we turn to the question why a class 2 prefix never stacks on top of a class 1 prefix, and a class 8 prefix never stacks on top of a class 7 prefix. Within Dèchaine et al.'s analysis, this must mean that the class 1 prefix and the class 7 prefix cannot be associated with Sort:

(96)

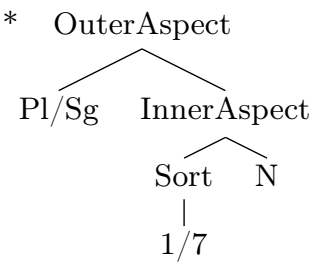

In fact, Dèchaine et al. postulate that these prefixes, as well as their plural counterparts only occur at $\mathrm{Pl} / \mathrm{Sg}$. Sort is taken to be empty whenever a prefixes in class $1,2,7$ or 8 appear:

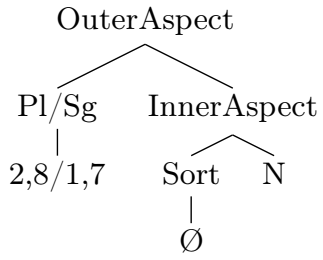

This is what they refer to as 'prefix-drop' in contrast to 'prefix-raising' which derives structures with a prefix in class $3,4,5$ or 6 at $\mathrm{Pl} / \mathrm{Sg}$ :

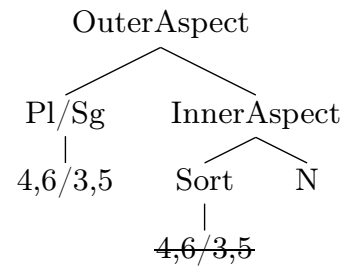

The question is why prefixes in classes $1,2,7$ and 8 behave differently from the prefixes in classes 3, 4, 5 and 6. A possible answer might derive from Dèchaine et al.'s claim that a prefix at Sort must 
classify a mass noun. Conceivably, the semantics of a prefix in class $1,2,7$ or 8 is incompatible with this. But some of Dèchaine et al.'s examples seem to contradict the claim that a prefix in Sort only applies to mass nouns or creates a mass denotation. Thus, in their (14) (p. 28), they give the analyses in (99) for the class 14 noun $u$-ta 'bow/bows' and the corresponding plural ma-u-ta 'bows':
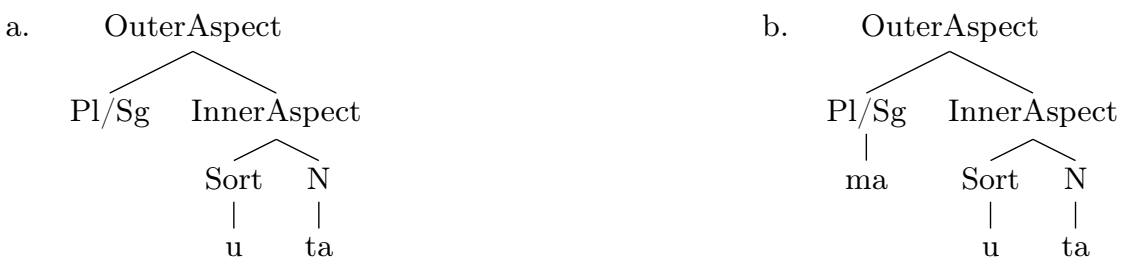

But it is somewhat unclear how 'bow' or 'bows' can be thought of as a mass noun and therefore also dubious that prefixes under Sort can be associated uniquely with mass readings. Correspondingly, there is no obvious reason in Dèchaine et al.'s framework why (100) should be excluded, hence no reason why another prefix should not appear at $\mathrm{Pl} / \mathrm{Sg}$ on top of a class 1 or class 7 prefix:

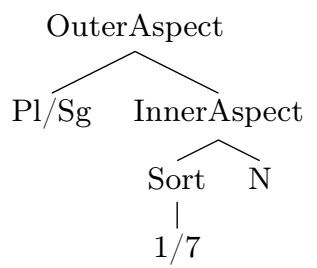

But under the analysis we presented in section 4.2, a prefix in class 2 or 8 cannot be added on top of a singular prefix in class 1 or 7 , since \# can be lexicalized together with the classifier $\mathrm{N}_{X}$ when $\mathrm{X}=1$ or 7 , and Specifier-formation is a last resort option. ${ }^{44}$

Crucially, our analysis provides a way of linking the stacking contrasts to the difference between classes 1 and 7 on the one hand and classes 3 and 5 on the other to the difference between classes 1,2 , 7 and 8 and classes 3, 4, 5 and 6 with respect to agreement with a conjunction of two singular nouns. By contrast, Dèchaine et al.'s proposal offers no way of understanding this link.

\subsection{Summary}

In this section, we have seen evidence for the following correlation in the Tsonga languages Changana and Rhonga: Whenever a conjunction of singular nouns in class X (classes 1 and 7) co-occurs with the same SC as nouns in the plural class $\mathrm{Y}$ (classes 2 and 8) paired with $\mathrm{X}$, a prefix in class $\mathrm{Y}$ cannot stack on top of a prefix in class X. Otherwise, stacking a plural prefix on a singular prefix is possible. We have also shown that this correlation is predicted by our analysis, which moreover accounts for the fact that while plural prefixes may stack on top of singular prefixes; we have found no cases where a singular prefix stacks on top of a plural prefix.

In addition, we have argued that our analysis of stacking is consistent with the Shona double plurals, provided the class 4 prefix $m i$ in Shona cannot lexicalize \#.

\section{All class prefixes are specifiers}

In section 3, we developed a specific analysis of the class 4 prefix $m i$ and the class 5 prefix $m a$ in order to account for the agreement patterns discussed in section 2 . This analysis claims that $m i$ and $m a$ lexicalize

\footnotetext{
44 We also note that $\mathrm{Pl} / \mathrm{Sg}$ instead of \# (only Pl) also allows singular prefixes on top of the stack.
} 
specifiers containing an $\mathrm{N}$ distinct from the overt noun and its extended projection. But in subsection 3.4, we pointed out that our theory of lexicalization entails that all plural prefixes must lexicalize specifiers of this kind, which led to the further conclusion that each singular prefix lexicalizes some classifier-like $\mathrm{N}_{X}$.

In this section, we will argue that this conclusion is independently supported by the relation holding between secondary and primary prefixes, and discuss the consequences of our conclusion with respect to the pairing of prefixes and nouns. We also show that even the agreement-markers on verbs, the SCs, must be taken to lexicalize a structure containing a classifier-like noun, as already proposed in section 3.5 and present independent evidence supporting this conclusion as well.

\subsection{Primary prefixes and secondary prefixes}

Analyzing the Bantu class prefixes as morphemes spelling out syntactic structures is at odds with a more traditional analysis, which would see Bantu nouns as morphological structures. But if prefixes are added to nominal stems in the morphological component, Bantu nouns must be considered to exhibit some highly exceptional properties. Usually, the prefixes are taken to be of two different types. The primary prefixes generally only occur on nouns of a particular noun class without affecting their meaning, e.g. $l i$ on class 5 nouns or si on nouns in class 7. A secondary prefix replaces or stacks on top of the primary suffix of a noun with a concomitant change in conceptual meaning. Thus, the Xhosa's $m$ is traditionally analyzed as the primary prefix of the class 1 noun $u$-m-ntu 'person', while bu in $u$-bu-ntu 'humanity' is considered a secondary prefix.

From a morphological perspective, the primary prefix of a noun is arguably analyzable as an inflectional affix of the noun spelling out its gender and number features, while a secondary prefix would be a derivational affix affecting both the meaning (like English -ness or -ity) and the gender of the derived noun (like German -keit). But then it comes a surprise that a secondary prefix can stack on top of a primary prefix, as, for example, in the form vu-mu-nhu 'humanity' related to mu-nhu 'person' in the Manyika dialect of Shona or the Shona form zi-banga 'big knife' with the augmentative prefix $z i$ added outside the primary class 5 prefix $R I$ (reflected only in the initial consonant: banga 'knife' ma-panga 'knives' - ma-zi-panga 'big knives'). ${ }^{45}$ Cross-linguistically, derivational affixes only occur inside inflectional affixes, a fact that most morphological theories seek to account for.

Another striking fact is that many prefixes occur both as secondary and primary prefixes. For example, $b u / v u$ is the primary prefix of class 14 nouns, e.g. $u$-bu-sika 'winter' in Xhosa, and Shona $R I$ is the primary prefix of nouns in class 5 , e.g. banga $=R I$-panga 'knife', as well as a secondary augmentative prefix with nouns in other classes, e.g. buka=RI-puka 'big beast' from the class 9 noun $m h u k a=N$-puka 'beast'. 46

The best way of understanding these properties of secondary prefixes is to analyze them as the inflectional/primary prefixes of silent nouns taking forms with secondary prefixes to be binominal constructs formed in the syntax. ${ }^{47}$ Then, forms with a secondary prefix on top of a primary prefix cease to be deviant with respect to what we know about the properties of complex words. In particular, they can be analyzed the same way as the stacking cases discussed in section $4 .{ }^{48}$ The meaning component added by

45 The Manyika data has been provided by Author 3 and is based on his fieldwork. The source for the Shona facts is Fortune (1955).

46 Lecarme (2002:4.4) observes similar facts in Somali, Yiddish and Breton (see also Stump (1989) and Acquaviva (2008)) and reaches conclusions that are similar to ours, but are implemented in a different way; cf. section 6.3 below.

47 Carstens $(1991,2008)$ also proposes that a secondary prefix is the primary suffix of a silent noun. We offer some comments on her proposal in subsection 5.2.

48 The non-stacking secondary prefixes must be merged as $\mathrm{N}_{X}$, while those that stack may occur in positions different from both $\#$ and $\mathrm{N}_{X}$, e.g. the degree 2 augmentative $z i$ in Shona discussed in the text, which precedes the class 5 prefix $R I$, but follows the class 6 prefix ma, which presumably is at \#. Thus we may also need an extra structural layer like Dèchaine et al.'s (2014) 'evaluative layer' mentioned in footnote 43. 
a secondary prefix will then come from the silent noun whose primary prefix it is, and the fact that for example $R I$ acts both as the primary prefix of class 5 nouns and an augmentative secondary prefix can be captured by saying that the silent noun giving rise to the augmentative reading is in fact in class 5 .

But if the noun $N_{X}$ inside the secondary prefix $R I$ is lexicalized by $R I$ in the way outlined in section 3 , the formal identity between secondary $R I$ and primary $R I$ can only be captured if the primary $R I$ contains $N_{X}$ as well. ${ }^{49}$ Thus, the augmentative $R I$ and the primary class 5 prefix $R I$ must contain exactly the same $N_{5}$ at the bottom, raising the question why $R I$ doesn't act as an augmentative with class 5 nouns.

To approach this question, we will draw on assumptions that will be discussed in a more general way in subsection 5.3. One possible answer might be that the combination of a noun and a primary prefix is always idiomatic in nature. Another possible answer would be that the exact meaning of the combination of a noun and a prefix is determined both by the meaning of the silent noun and the meaning of the overt noun. For example, adding $N_{5}$ (contained in $R I$ ) might have the effect of selecting entities with certain fixed properties from the denotation of the overt noun, and it is conceivable that while these properties are conventionally associated with all class 5 nouns, they are perceived as aberrant with respect to the standard properties of members of other classes. It is noteworthy in this connection that the semantic impact of the secondary $R I$ is much less precise than the term 'augmentative' might suggest: $R I-N_{x}$ (with $x \neq 5$ ) can mean 'ugly, unshapely N' in addition to 'big N'.

Either way, we will expect that there is no way of substituting an augmentative $R I$ for the primary class 5 prefix $R I$, although augmentative $R I$ can be substituted for the primary prefix in the other classes. This prediction cannot be tested just by looking at forms like banga $=R I$-panga 'knife' (class 5 ), since presumably $R I$-panga with primary $R I$ denotes knives of different sizes subsuming the meaning of the augmentative. But Shona has a second augmentative $z i$ which merges on top of augmentative $R I$ to derive the meaning 'exceptionally big', acting as a 'degree 2 augmentative' in Fortune's (1955) terms. Thus: $m h u k a=N$-puka 'beast' (class 9) - buka =RI-puka 'big beast' $-z i$-buka $=z i$-RI-puka 'exceptionally large beast'. So, if augmentative RI could replace the primary class 5 prefix $R I$ as well, we should have $z i$-banga $=z i$-RI-panga with augmentative $R I$ and $z i$ adding an extra degree of 'bigness' to the one already introduced by augmentative $R I$. But according to Fortune (1955:104-105), zi-banga only has the 'degree 1' reading 'big knife' and not also the degree 2 reading 'exceptionally big knife', and all class 5 nouns behave the same way as banga. Therefore, we conclude that an augmentative secondary $R I$ cannot in fact be substituted for the primary class 5 prefix $R I$, and explain this by saying that the two are in fact the same.

We would now like to emphasize that since secondary prefixes are commonly analyzed as being associated with a silent $\mathrm{N}$, saying that secondary $R I$ is identical to primary $R I$ presupposes that primary $R I$ is also associated with a silent $\mathrm{N}$, precisely as we first claimed that all class prefixes are, in section 3.4. In section 3.4, this conclusion was seen to be forced by specific theoretical assumptions, i.e. the theory of lexicalization in section 3.3 and the one-to-one correspondence between features and syntactic heads. Therefore, those specific theoretical assumptions ultimately predict the impossibility of substituting secondary $R I$ for primary $R I$, and the correctness of this prediction validates them over competing approaches that would not have led to this prediction, e.g. the 'spanning' approach mentioned in footnote 28.

This type of 'blocking' of a secondary prefix is not limited to class 5. Carstens $(1991,2008)$ shows that in Swahili no primary prefix can be replaced by a secondary prefix of the same class, and proposes an explanation for this, to which we now turn.

\footnotetext{
49 A similar conclusion is reached by Hall (2015) for what we might call primary and secondary classifiers in Chinese.
} 
5.2 Carstens (1991, 2008)

Like us, Carstens $(1991,2008)$ claims that secondary prefixes are connected to silent nouns. Unlike us, however, Carstens does not extend this analysis to the primary suffixes. In fact, she explicitly argues against such an extension. The argument is based on the observation that although the class 7 prefix is used as a diminutive secondary prefix in Swahili, and the class 5 prefix is used as an augmentative secondary prefix, substituting the secondary class 7 prefix for the primary prefix of a noun in class 7 or the secondary class 5 prefix for the primary prefix of a class 5 noun has no effect on the meaning. ${ }^{50}$ This is precisely the observation we discussed for Shona in the preceding subsection.

However, Carstens' conclusion from this follows only if one must assume that the nouns hidden inside the secondary prefixes must have the same semantic effect on any noun the prefix is added to. In the preceding subsection, we denied this and proposed an alternative view consistent with our claim that the same noun occurs inside both secondary and primary prefixes of the same class. We also showed how this explains why one cannot obtain an augmentative reading by adding an augmentative class 5 prefix to a class 5 noun.

To account for the same fact, Carstens must actually add an auxiliary assumption, since otherwise nothing would prevent a class 5 noun from combining with her augmentative silent noun to create a structure where the class 5 noun, with the usual class 5 prefix, would in fact have an augmentative reading on her account. To this end, she initially invokes what she calls 'Blocking by Homonymy' (Carstens 1991: 222 ) intended to apply under word formation:

Blocking by Homonymy (BBH)

$* \alpha, \alpha$ a derived word, where $\alpha$ duplicates the form of an existing lexical item $\beta$.

However, she subsequently notes that 'BBH thus achieves descriptive adequacy, but lacks motivation as a general condition on Kiswahili words, and is therefore anomalous in its inclusion among the WFCs [Word Formation Conditions].' (p. 233). Consequently, she proposes to view (101) as a reflex of the general condition in (102), crucially relying on the characterization of identification in (103) (Carstens 1991: 233-234): $:^{51}$

(102) Zero-morpheme Licensing Principle:

Zero-morphemes must be identified.

(103) Zero-morpheme Identification Condition:

A zero-morpheme is identified if and only if its features are unambiguously represented on the derived word.

To assess the impact of (103), it is necessary to determine what exactly 'unambiguously represented' should mean in the context of Carstens' analysis. Crucially, she assumes that (103) must apply at the point where the covert noun has been affixed to the host, but the number head hosting the prefix has not yet been added (Carstens 1991: footnote 16, p. 233). This entails that 'unambiguously represented' cannot in general be understood to require a difference in the phonological form of the affix (the silent $\mathrm{N}$ ), since then (103) would apply to the licit form in (104-a) (with $x \neq y$ ) as well as to (104-b), which it is intended to exclude:

a.

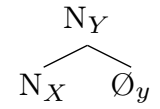

b.

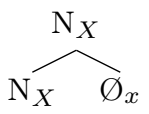

50 Although it is actually unclear how one can know this, since, for example, class 7 nouns presumably denote both big and small things.

51 The connection with other proposals in the literature is rather tenuous, as noted by Carstens in her footnote 16, p. 233. 
Rather, Carstens suggests that the gender feature $x$ associated with the covert noun is unambiguously represented in the structure only if the gender feature on the noun derived by affixation can unambiguously be traced back to the zero noun. This clearly holds in (104-a), but, according to Carstens, not in (104-b), where the host noun $\mathrm{N}_{X}$ is an alternative source for the gender feature $x$ associated with the derived noun.

Notice, however, that the association of the gender feature $y$ with the derived noun in (104-a) is taken to reflect a structural property of these constructs: The derived noun inherits the gender of the affixed zero noun, because the latter is taken to be the head of the derived noun. But assuming that (104-a) and (104-b) have the same structure, the zero noun must then be identifiable as the head of (104-b) as well, and since feature-percolation to the root node must originate from the head, the gender feature of the derived noun is unambiguously traced back to the zero noun in (104-b), too. Hence, (102)-(103) does not provide a coherent way of accounting for the lack of an augmentative reading with class 5 nouns, given that (102)-(103) must apply by inspecting structures at the level of derivation corresponding to (104-a)-(104-b).

We acknowledge that (102) itself and also the 'Morphological Bijection Requirement' proposed subsequently (Carstens 1991: 236) are a priori perfectly plausible candidates for being general well-formedness conditions. The problem is that their applicability within Carstens' analysis rests on (103), which, as we have just seen, cannot be coherently applied so as to derive Carstens' conclusion (Carstens 1991:236): 'A diminutive interpretation for a group D [class 7] noun, for example, will involve a failure to supply a morpheme in the word corresponding to the diminutive meaning.' By contrast, this conclusion is immediate on our analysis in subsection 5.1, which posits no difference between secondary prefixes and the corresponding primary prefixes of the same class, but makes the semantic impact of the prefix contingent upon the semantics of the host noun.

\subsection{Combinatorics}

On the account we are developing, the fact that any given overt noun only combines with a proper subset of all the available singular class prefixes, cannot be captured by saying that the choice of a class prefix is determined by transmission of gender features from the overt noun itself. Indeed, the overt nouns might as well be taken to be genderless, as we have already pointed out.

Instead, the combinatorial possibilities must be restricted either by idiom formation or by a general semantic requirement to the effect that (the extended projection of) the silent noun must be compatible with the overt noun in a sense to be made more precise. This conclusion converges with the traditional view that each class has a core meaning associated with it, but also contains a number of nouns whose semantics is not readily consonant with the core meaning. The latter may be listed in the lexicon as phrasal idioms while the core meaning will be taken to be a consequence of the meaning of the silent noun inside the class prefix composing with the overt noun. This view is similar to Hall's (2015) conclusions for the Chinese classifiers based on contrasts like (105) (Hall (2015:167)):

$$
\begin{aligned}
& \text { a. san-zhi hua } \\
& \text { three-classifier flower } \\
& \text { 'three flowers (long on their stalks)' }
\end{aligned}
$$

b. san-duo hua

three-classifier flower

'three flowers (round, with focus on floweriness)'

The choice of classifier determines the meaning. (We return to Hall's analysis of the Chinese classifiers in section 6.5.)

Relatively clear examples of idiomatic formations include Xhosa class 7 nouns like $i$-si-kolo 'school' and $i$-si-talato 'street'. These have meanings that don't fit the core meaning of class 7 , hence not the meaning of $N_{7}$, but are probably in class 7 just because reanalyzing the initial $s$ of Afrikaans skool and straat as part of the class 7 prefix si provides a way of making these loanwords consistent with 
the phonotactics of Xhosa. ${ }^{52}$ In this case, the meaning of the combination of $N_{7}$ with the root is noncompositional.

At the other end of the specter, we have nouns formed with the class 1 prefix or the class 2 prefix. With the exception of some nouns in class $1 \mathrm{a} / 2 \mathrm{a}$, e.g. $u L$ 'the letter L' / ooL 'the L's', all nouns are interpreted as denoting human beings in combination with the class 1 prefix and the class 2 prefix. This suggests a compositional meaning based on the meaning of the $N_{1}$ contained both in the class 1 prefix and the class 2 prefix, e.g. 'person' (overridden by an idiomatic meaning in the case of $u L$ etc.).

From this perspective, the class prefixes act as modifiers. For example, we might assign them meanings conforming to the template $\lambda P \lambda x . \llbracket N \rrbracket(x) \& P(x)$, where $\llbracket N \rrbracket$ is the denotation of the noun contained in the prefix. Then, if the silent noun means 'person' in class 1 , the class 1 prefix would have the meaning $\lambda P \lambda x$.person $(x) \& P(x)$ which combines with the meaning of fazi 'female', for example, to give $\lambda x . p e r s o n(x) \&$ female $(x)$, i.e. 'woman'. 53

Even without providing a detailed account of all the class prefixes based on the meanings of the silent $N_{\mathrm{s}}$ assumed, we think this line of analysis is plausible. After all, there are classifier languages, and we essentially propose treating Bantu prefixes in whatever way is optimal for classifiers in those languages. As we will see in section 6.5, the properties of the Bantu class prefixes that have led us to this analysis, correspond fairly well to properties of Chinese classifiers that Hall (2015) uses to motivate his analysis of them. Therefore, we shall take it that each class prefix contains a specific $N$ that acts as a classifier with respect to the noun the prefix combines with. By this we mean that the $N$ inside the prefix essentially modifies the overt noun and thereby picks out a specific proper subset of all the things the overt noun may denote, unless a particular prefix + noun combination is listed in the lexicon and assigned an idiomatic meaning.

\subsection{Secondary SCs and the nature of agreement}

In section 3.5, we showed that our analysis of class prefixes leads to the conclusion that SCs also lexicalize Specifiers built on some classifier $\mathrm{N}_{X}$ :

$$
\text { a. } \overbrace{\mathrm{N}_{X} \mathrm{~V}}^{\text {Singular } \mathrm{SC}}
$$

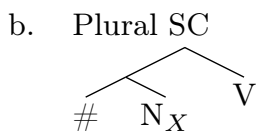

This conclusion leads to an expectation that does not arise on analyses identifying SCs merely with collections of gender and number features. On such analyses, there is no room for 'secondary SCs' analogous to the secondary prefixes on nouns discussed above, if a secondary prefix is always associated with a silent $\mathrm{N}$ responsible for the meaning change it induces, as argued both by Carstens (1991, 2008) and by us. But the analysis in (106) does provide room for the required silent N, i.e. $\mathrm{N}_{X}$. More precisely, an SC may behave like a secondary SC when the $\mathrm{N}_{X}$ in it does not copy the $\mathrm{N}_{X}$ in the class prefix on the subject.

The expectation that arises from this seems to be met. We can see this by considering a fact about Changana. As in other Bantu languages, a class 1 subject co-occurs with SC1 ( $a$ in Changana), and a class 2 subject comes with $\mathrm{SC} 2$ :

\footnotetext{
52 Most other loan words go into class 9.

53 Likewise, the $N$ inside the class 9 prefix might denote something like living things: Class 9 is sometimes referred to as 'the animal class', but also contains a few nouns denoting human beings,e.g. $i$ - $n$-doda 'man', and plant names in addition to nouns that must combine with the class 9 prefix in an idiosyncratic fashion, e.g. $i$ - $n$ - $d l u$ 'hut'.
} 

a. B'ava a-le
nay.
1.father SC1-eat.PF 9.meat
'Father ate meat.'
b. Va-b'ava va-le nay. 2-father SC2-eat.PF 9.meat 'The fathers ate meat.'

(Changana)

But it is also possible to use SC7 and SC8 to convey contempt or disapproval of the person(s) denoted by the subject:
a. B'ava xi-le nay.
1.father SC7-eat.PF 9.meat
'Father ate meat.'

b. Va-b'ava svi-le nay. 2-father SC8-eat.PF 9.meat 'The fathers ate meat.'

(Changana)

This seems to reflect the use of the class 7 and class 8 prefixes as 'secondary prefixes' on nouns with the same semantic effect. As secondary prefixes, $x i$ and svi combine with nouns of all noun classes inducing a derogatory reading. In accordance with the analysis of secondary prefixes in subsection 5.1, a secondary $x i$ and svi must lexicalize a structure containing a noun which is the source of the semantic effect:
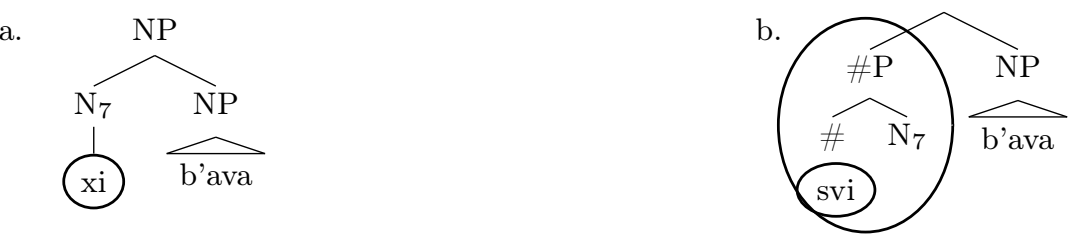

The SCs in (108) cannot acquire a gender feature by copying the gender of the subject. Rather, any gender feature associated with the SC would have to come from a class 7 noun lexicalized by the SC itself, in fact the same $N_{7}$ that occurs in (109): ${ }^{54}$
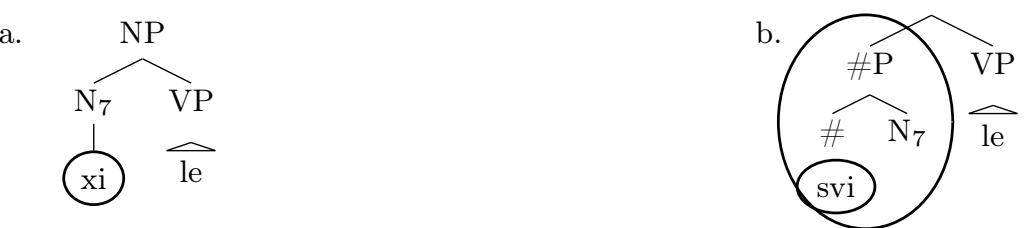

In subsection 5.1, we argued that the semantic effects of using a prefix of some class $X$ as a secondary prefix on a noun in a different class $Y$ must arise from the fact that the $\mathrm{N}_{X}$ inside the prefix denotes entities with a property not shared by all things denoted by nouns in class $Y$. Similarly, the fact that a SC can be used as a 'secondary SC' imparting a special property to the subject noun phrase, as in (108), must be due to the $\mathrm{N}_{X}$ inside the SC denoting entities with a property not uniformly shared by the entities in the denotation of nouns in the noun class that the subject belongs to. That is, we must say that subject/verb agreement in a language like Changana is essentially semantic agreement: The $\mathrm{N}_{X}$ inside the SC associates a certain set of properties with the individuals that the predicate applies to. ${ }^{55}$

In subsection 5.1, we also argued that the 'blocking' of a secondary prefix in class $X$ with nouns in class $X$ must be understood as evidence that the secondary prefix of class $X$ is in fact the same as the primary prefix of class $X$. In particular, they contain the same $\mathrm{N}_{X}$, but the properties associated with

\footnotetext{
54 From this point of view, the SCs in (108) are comparable to epithets like 'the bastard(s)' in You know my father. The bastard always wants meat. However, the sentences in (108) do not display properties consistent with analyzing them as sentences with a dislocated subject co-occurring with a covert resumptive subject pronoun in class 7 or 8 mirroring Father, the bastard ate the meat.

55 In this sense, we agree with Carstens (2011) that subject/verb agreement in Bantu does not involve valuation of unvalued uninterpretable features.
} 
things in the dentation of $\mathrm{N}_{X}$ are conventionally taken to be common to all things in the denotation of a class $X$ noun. This now carries over to the analysis of SCs. For example, the fact that SC7 $x i$ and SC8 svi in Changana do not give rise to a derogatory reading when the subject is in class 7 or 8 , follows from the assumption that $x i$ and svi used as 'primary SCs' contain the same $\mathrm{N}_{7}$ as the 'secondary SCs' in (108), but the property of things denoted by $N_{7}$ that gives rise to the semantic effect seen in (108), is conventionally shared by all individuals denoted by a noun in class 7 or 8 .

At this point, one may as well adopt the most general conclusion consistent with our findings: All SCs (not only those that are also used as 'secondary SCs;) contain an $\mathrm{N}_{X}$ just like the corresponding class prefixes on nouns.

From this point of view, the only difference between (107) and (108) is that the $\mathrm{N}_{X}$ in the SC copies the $\mathrm{N}_{X}$ in the class prefix of the subject in (107), but not in (108). We will assume that the option of copying the $\mathrm{N}_{X}$ in the subject's prefix - now the residue of what we called formal agreement in section 2 - is taken unless a special semantic effect is intended, as in (108), or unless the option cannot be taken for grammatical reasons, as when the subject is a conjunction of nouns with different classifiers or there is no prefix that can lexicalize a $\# \mathrm{P}$ with the $\mathrm{N}_{X}$ copied from the conjoined nouns at the bottom as in the cases examined in sections $2.5-2.6 .^{56}$

The default options chosen in the latter case, i.e. SC2 ba for human nouns and SC8 zi elsewhere, are plausibly just instances of what we see in (108). The choice of SC is again based on semantic properties of the $\mathrm{N}$ it contains. We acknowledge, however, that an important question remains to be answered: Why aren't SC3 $i$ and SC6 $a$ also used as default SCs? ${ }^{57}$ We submit that this is because defaults must be the least richly specified members of a set of elements, and that the nouns inside SC2 and SC8 are less specified than the nouns in SC4 and SC6, i.e. the semantic content of $\mathrm{N}_{1}$ may be just 'human' and that of $\mathrm{N}_{7}$ just 'thing'. Although we are not as yet able to provide a detailed semantic analysis to support this claim, we think that it will ultimately provide a way of explaining a striking correlation: The default SCs correspond to prefixes that are never used as secondary prefixes of the sort discussed here and in section 5.1, nor to form irregular plurals like those discussed in 2.1 and $2.4 .^{58}$

Alternatively, one might take nouns with a class 4 or class 6 prefix to denote a different type $\mathrm{Z}$ of pluralities than a conjunction of two singular nouns. If SC4 and SC6 make the verbal predicate applicable only to pluralities of type Z, it would then follow both that SC4 and SC6 don't occur as default SCs with conjoined singular subjects of different classes, and that SC4 and SC6 don't even occur when the subject is conjunction of two class 3 noun or two class 5 nouns. ${ }^{59}$ By contrast, SC2 and SC8 must make the verbal predicate applicable to the type of pluralities denoted by conjunctions of singular nouns, and, correspondingly, nouns with a class 2 or class 8 prefix must denote pluralities of the same type as conjunctions of singular nouns.

However, arguments developed in Schwarzschild (1996) make this line of analysis less appealing, and more importantly, Xhosa and the other languages discussed here provide no independent evidence that the pluralities denoted by class 4 and 6 nouns are any different from those denoted by class 2 and class 8 nouns. For example, both classes of plural nouns combine with numerals counting individuals, excluding

56 Unlike Xhosa, some languages might not have the option of using formal agreement (as conceived of here) with conjunction of singular nouns. The responses of a group of Zulu consultants (Author 1 and Author 2's field work) differ sharply from those obtained from our Xhosa consultants when it comes to agreement with two nouns in class 1 or class 7 or 9 . If the conjoint nouns were human, then the chosen SC marker was the class 2 ba (clearly also for class 7 and 9 nouns). If the conjoint nouns were inanimate, then the agreement marker was class 15 . (The native speakers felt rather uneasy when presented with a conjunction of inanimate nouns from class 1 : half of the speakers opted for class 2, the other half chose class 15.) If the conjoint nouns were a mixture, i.e. one animate, one inanimate, the answers were a mixture of SC2 and SC15.

57 A reviewer raises this question.

58 The derogatory effect of using SC8 in (108-b) is different from that associated with the secondary class 5 prefix $R I$ in Shona (discussed in 5.1). In (108-b), this effect most likely arises from the $\mathrm{N}_{7}$ inside SC8 denoting 'things' as opposed to humans. If so, we correctly predict that $x i$ and svi are only employed as derogatory secondary prefixes with nouns denoting human beings.

59 But a conjunction of two plural nouns in class 4 or class 6 must then denote the same type of plurality as a single noun with a class 4 or class 6 prefix; see footnote 18. 
the possibility that class 4 and class 6 nouns denote 'collectives' or 'groups' or are in some way more mass-like (as might be suggested by the existence of class 6 mass nouns like $a$ - $m a-n z i$ 'water') than plurals in class 2 or 8 .

\subsection{Summary}

What we have seen is this section, is that the formal analysis adopted to explain the properties of nouns in class 4 and class 6 leads to the further conclusion that all class prefixes and SCs correspond to structures with a $\mathrm{N}$ at the bottom. Since the gender-features determining the shape of the prefix/SC must come from this $\mathrm{N}$, the pairing between prefixes and (overt) nouns cannot depend on the gender-features of the (overt) noun, but must depend on a semantic relation between the silent $\mathrm{N}$ and the overt noun or idiom formation. In this sense, our analysis is closer to traditional accounts of the Bantu noun classes than to the generative tradition initiated by Carstens (1991).

\section{The broader perspective}

We have so far only discussed nominal structures in (a small sample of) Bantu languages. But the theoretical assumptions we have adopted lead to the expectation that prefixes with similar properties to the Bantu class prefixes examined here should be analyzed in a similar way in other languages as well. In this section, we look at some analyses of languages outside the Bantu group (Manjaku, Somali and Chinese) and argue that the claims made in these analyses as well as the empirical facts used to motivate them give us some reason to believe that this expectation may well be fulfilled.

6.1 The argument for analyzing Bantu class prefixes as specifiers

On the empirical side, our claim that every class prefix in Bantu is a phrase sitting in a specifier-position in the host noun's spine originates from the observation that the shape of a plural class prefix cannot in general be taken to be determined by a gender feature inherited from the host noun. Rather, if gender is relevant at all, the gender associated with a plural prefix must originate from a silent noun different from the host. Therefore, the relevant plural prefixes correspond to structures comprising the three heads \#, G and N, with N silent.

Specific theoretical assumptions entered the picture, when we considered the question why the $\mathrm{N}$ providing the gender of the prefix is silent. Our answer was that the prefix in fact lexicalizes all the three heads \#, G and $\mathrm{N}$ in accordance with a specific theory of phrasal lexicalization, as outlined in section 3.4. But this theory allows a single morpheme to replace a non-singleton set of heads only if those heads form a constituent in the syntactic tree. This in turn excludes the structural analysis in (111), where the silent noun would be a sister of the host noun $\left(\mathrm{N}_{X}\right)$ :

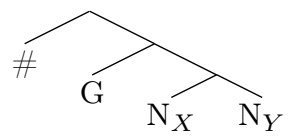

The only remaining possibility is then (112), where [ \# [ $\left.\left.\mathrm{G}\left[\mathrm{N}_{Y}\right]\right]\right]$ occurs as a specifier:

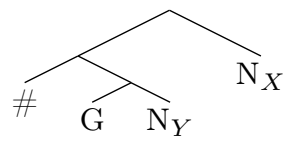


But if lexicalization only applies to constituents, and, moreover, each head carries a single morphosyntactic feature, we also must conclude that no portmanteau morpheme lexicalizing both gender $(G)$ and number (\#) should be able to occur as a prefix on $\mathrm{N}$ with the structure in (113):<smiles>[Te]CC[Te]</smiles>

The alternative in (114), however, is not a licit structure, since nominal functional heads only project on the spine of a noun: ${ }^{60}$

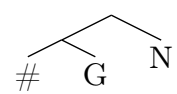

The fact that the Bantu prefixes nevertheless look like portmanteau morphemes (for gender and number), therefore forces the conclusion that all Bantu class prefixes correspond to constituents with a separate $\mathrm{N}$ at the bottom, as in (112). Thus, our specific theoretical assumptions actually predict the existence of the empirical facts that we have used to motivate the analysis in sections 4 and 5 .

This prediction does not arise on a 'spanning' approach to lexicalization which would allow a single morpheme to lexicalize a set of terminals that do not form a constituent. A portmanteau prefix $M$ would then be able to lexicalize \# and $\mathrm{G}$ on the projection line of the host $\mathrm{N}$ in [ \# [ G [ N ]]] leaving $\mathrm{N}$ to be lexicalized by a different morpheme. Therefore, it would not by itself explain the facts indicating that class prefixes contain an extra $\mathrm{N}$ in Bantu. Nor would it make the more general prediction discussed in the following subsections.

\subsection{A related proposal}

A more general consequence of our theoretical assumptions is that nominal portmanteau prefixes in any language should have the internal structure we ascribe to the Bantu class prefixes. In particular, they should contain an $\mathrm{N}$ distinct from the overt noun hosting the prefix and have specific syntactic and semantic properties stemming from this.

We are obviously not in a position to tell to what extent this prediction is borne out. But we want to mention ideas by Kihm (2005) that seem promising from our perspective.

Kihm's investigation of Manjaku (Niger-Congo) reveals that the noun class prefixes in this language have some properties typical of derivational morphemes in a way reminiscent of Bantu. Thus, the choice of class prefix may determine the meaning of the prefix + noun combination as exemplified by (115), with lik taken to roughly mean 'water' (Kihm 2001: 11)
a. pë-lik
9-lik
b. $\quad$ m-lik
6-lik
c. ka-lik
7-lik
'fruit juice'

Also, the pairing of singular and plural classes is subject to exceptions reminiscent of Bantu. For example, many nouns in class 9 have plurals in class 8 rather than class 10.

Kihm also notes that these properties are not generally found with number/gender markers in Romance.

Kihm then suggests that the special properties of the Manjaku class prefixes may be explained by taking them to spell out $n$ analyzed as 'a root, i.e. a member of the lexicon, and that to the difference of all other roots, except $v$, it is endowed with a category, namely $\mathrm{N}$ " adding that ' $n$ is a label for the set of prototypical nouns, or protonouns, each having its own meaning and exponent'. Kihm's 'little n' differs

\footnotetext{
60 In this section, the gender head $\mathrm{G}$, seen to be redundant in our account of Bantu, is retained for ease of exposition.
} 
from the way 'little n' is generally characterized in the literature and is more similar to our 'silent' Ns. The various Ns we claim occur inside the Bantu class prefixes can in fact be viewed as protonouns in Kihm's sense.

With respect to the contrast between Manjaku and Romance, Kihm makes another proposal which is interesting in relation to our analysis of Bantu. He points out that the gender/number markers in Romance differ from the Manjaku class prefixes both with respect to their semantic properties and their morphosyntactic status: The Romance gender/class markers typically lack the properties of derivational morphology exhibited by the Manjaku class markers, and they are also suffixes rather than prefixes. Kihm relates these two properties in the following way: The categorizing head 'little n' spelled out by the Romance gender/number markers is not a lexical root, and therefore the Romance gender/number markers cannot affect the meaning of host nouns the same way the Manjaku class prefixes can. On the other hand, taking the Romance $n$ as a purely functional head accounts for the fact that it spelled out by a suffix, if, as Kihm asserts, a purely functional $n$ always attracts N. Actually, he takes this a little further by saying that an $n$ containing a lexical root, as in Manjaku, cannot attract $\mathrm{N}$, hence must be a prefix. But as we shall see in the next subsection, this may not be quite right.

\subsection{Suffixes may also be specifiers}

We think that ' $\mathrm{n}$ containing a lexical root (a protonoun)' (using Kihm's terms) would actually also be an appropriate characterization of the class suffixes in Somali by the criteria Kihm applies to Manjaku. To account for gender change between singular and plural suffixes (so-called 'gender polarity'), Lecarme (2002:109) in fact analyzes the Somali plural suffixes in a way highly reminiscent of Kihm's characterization of the Manjaku class prefixes (as well as Schadeberg's (2001) and our own characterization of the Bantu class prefixes): 'Plural affixes are nominal categories that behave like other nominal affixes with a fixed inherent gender.'

$$
\begin{aligned}
& \text { a. } \operatorname{libáax}_{M} \text { 'lion' - libaaxyó } F \text { 'lions' } \\
& \text { b. } \text { goól }_{F} \text { 'lioness' - gooló } M \text { 'lionesses' } \\
& \text { c. } \text { sheekó }_{F} \text { 'tale' - } \text { sheekoóyin }_{M} \text { 'tales' }
\end{aligned}
$$

In Kihm's terms, this suggests that a 'a little n' containing a lexical root is represented as a suffix in Somali.

Furthermore, Lecarme (2002:128) suggests that due to the less than purely functional nature of [ Num $[n]$ in Somali, 'a particular requirement about what constitutes a morphologically well-formed noun in the language blocks the syntactic joining of $\mathrm{N}$ and Num, leaving a stranded Num morpheme.' This is intended to account for the fact that the combination of the host noun and the suffix lacks characteristic word-like properties. However, attributing this to a morphological well-formedness condition seems at odds with Lecarme's subsequent affirmation that the plural suffix undergoes 'morphological merger' with the host noun.

Within our set of assumptions, an alternative account of Somali emerges. We may say that the Somali plural suffixes lexicalize specifiers of the same type that are lexicalized by class prefixes in Bantu and, we would submit, in Manjaku:

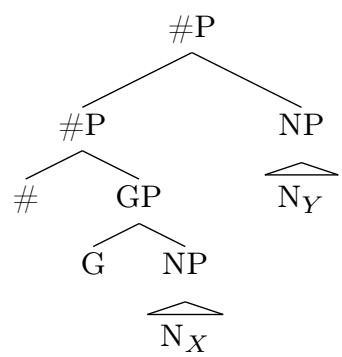


In this structure, the host noun $\mathrm{N}_{Y}$ cannot adjoin to the protonoun $\mathrm{N}_{X}$ or the whole specifier for perfectly general syntactic reasons. Nor can the protonoun or the specifier adjoin to the host noun. This corresponds to Kihm's assertation that $n$ containing a lexical root cannot attract N. But nothing will prevent the NP headed by the host noun $\mathrm{N}_{Y}$ from raising to a higher specifier-position giving (118):

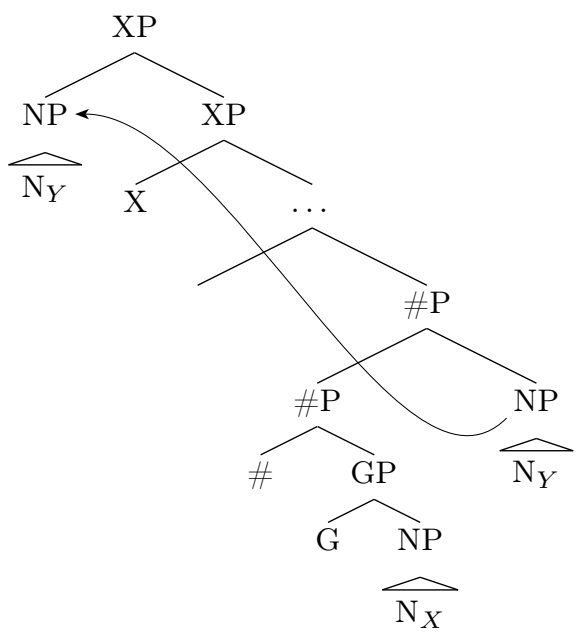

In (118), the syntactic relation between $\mathrm{N}_{X}$ and the specifier lexicalized by the suffix is such that the absence of the relevant word-like properties noted by Lecarme becomes understandable.

\subsection{Somali vs. Romance}

As already mentioned, Kihm (2001) notes that the gender/number-marking suffixes found in Romance do not typically exhibit the properties that motivate saying that $n$ contains a lexical root (protonoun) in Manjaku. Hence, he concludes that $n$ must be purely functional in Romance and also proposes that a purely functional $n$ must attract the head noun and therefore must end up as a suffix. Again, our theoretical assumptions suggest an alternative.

In our analysis of Bantu, Kihm's protonoun is represented as an $\mathrm{N}_{X}$, the closest counterpart to his $n$, packed together with $\mathrm{G}$ and the number head \# inside a specifier on the host noun's extended projection line:

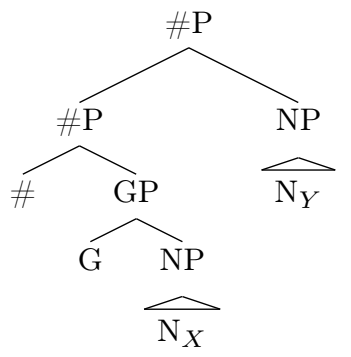

From this perspective, saying that $n$ does not contain a protonoun in Romance, corresponds to saying that only \# and G are added on top of the host noun, not also the $\mathrm{N}_{X}$ occurring in (119). However, since \# and G must be on the extended projection line of some N, the structure in (120) is not licit: 
$(120)$

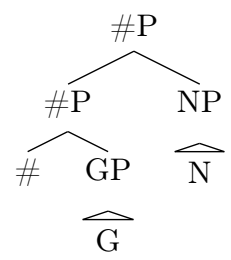

Therefore, \# and G cannot be inside a specifier on the host noun's projection line, when there is no protonoun corresponding to $\mathrm{N}_{X}$ in (119), and the structure of Romance nouns must instead be like (121):

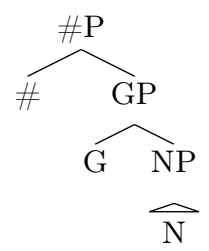

But in (121), \# and G do not form a constituent and therefore cannot be spelled out by a single portmanteau morpheme. Yet, the gender/number suffixes are in fact portmanteau morphemes in some Romance languages, e.g. Italian:

$\begin{array}{lll} & \text { singular } & \text { plural } \\ \text { masculine } & -o & -i \\ \text { feminine } & -a & -e\end{array}$

If, however, suffixation results from movement of the NP across \# and G, (121) gives rise to (123). ${ }^{61}$

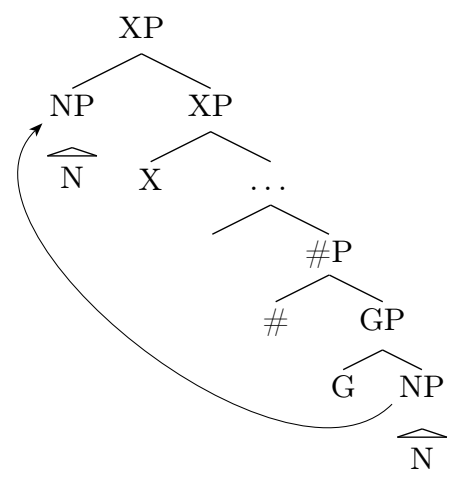

As already mentioned in sections 3.4 and 4.2 (footnote 37), Pantcheva (2011) and Starke (2009, to appear) argue that traces (unpronounced lower copies) are not visible to the lexicalization procedure so that (123) is equivalent to (124) when lexicalization applies:

\footnotetext{
61 A fact about the Colonnata variety of Italian is consistent with adopting (121) for Italian: In this variety, feminine nouns ending in $-a$ in the singular have plurals in $-y a$, where $-y$ - is plausibly identified with the plural morpheme $i$ also occurring in masculine nouns, i.e. the spell-out of \#, and $a$ corresponds to G, as in the singular feminine forms; cf. Manzini and Savoia (2005: III, 618 ff), Taraldsen (2009). The Colonnata variety can be analyzed exactly like Standard Italian except that it lacks the portmanteau -e.
} 
$(124)$

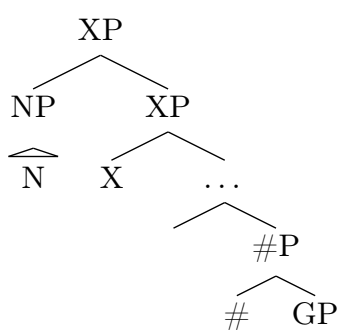

If this is correct, movement of the NP creates a structure where \# and G do form a constituent and accordingly can be replaced by the morphemes in (122).

If the number head \# is universally higher than the gender head G, as seems plausible, Spanish provides independent evidence for NP-movement. In Spanish, gender and number are marked by distinct morphemes:

$\begin{array}{lll} & \text { singular } & \text { plural } \\ \text { masculine } & -o & -o-s \\ \text { feminine } & -a & -a-s\end{array}$

But the gender-marker precedes the number marker. This suggests a derivation akin to those posited for $N$-A-Num in Cinque's (2005) account of Greenberg's Universal 20 (ignoring traces):
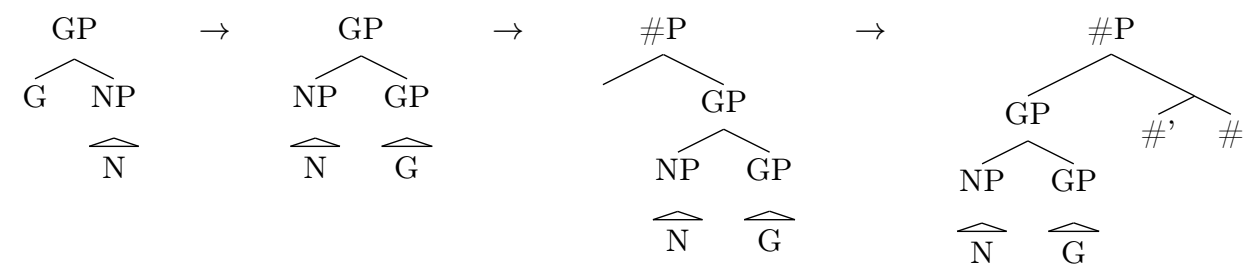

In the resulting structure, $o / a$ will lexicalize $\mathrm{G}$ and $s$ will lexicalize \#. (But \# and $\mathrm{G}$ could not be lexicalized by a single portmanteau morpheme, as in Italian.)

Thus, it seems that the general assumptions we have adhered to in our analysis of the Bantu class prefixes, also allow us to accommodate class suffixes of the Somali variety and the Romance gender/number suffixes in a way consistent with Kihm's and Lecarme's empirical observations. That is, our assumptions predict that class suffixes may contain a protonoun, as in Somali, but need not do so, as in Romance. ${ }^{62}$ But class prefixes must contain a protonoun, as in Bantu and Manjaku.

\subsection{Chinese}

In Chinese nouns may be preceded by classifiers. In his study of Chinese classifiers, Hall (2015) concludes that the classifiers spell out phrasal structures standing in a Spec/head relation to a projection of the host noun. Interestingly, Hall reaches this conclusion on the basis of theoretical assumptions that differ from ours, but nonetheless his account ends up converging with ours in crucial respects.

Hall's analysis is grounded in a theory of syntactic structure and lexicalization emanating from Brody (1997, 2000) and further developed by Adger (2013). Syntactic structures are represented in the form of 'telescoped' trees. Thus, classical X-bar structures like the trees in (127) correspond to trees like (128)), where the nodes $\mathrm{X}, \mathrm{Y}$ and $\mathrm{Z}$ represent phrasal projections and their heads at the same time:

\footnotetext{
62 This is not to say that class suffixes never contain a protonoun in Romance. In Italian, agreement with a conjunction of singular masculine nouns shifting to feminine in the plural leads to masculine plural inflection on adjectives and participles, as noted in section 2.4. This is similar to what we saw with Bantu nouns with unexpected class 6 plurals and should lead to the conclusion that the $a$ in forms like le dita 'the fingers' actually spells out a structure containing a (feminine) protonoun.
} 
a.

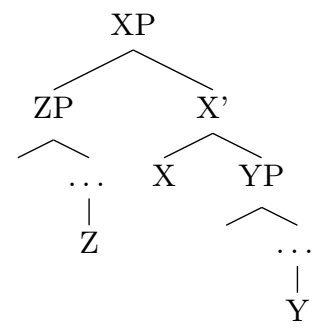

b.

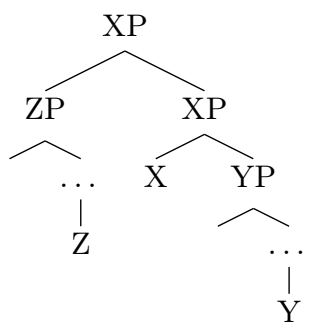

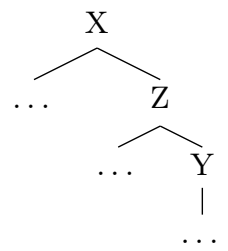

By convention, a left daughter of $X$ is a specifier of $X(P)$, while a daughter on the right is its complement. $X$ in (128) may be either a lexical head embedding a complement or a functional head on the extended projection line of $Y$ (or some lower head).

An ordered set of heads meeting the requirement set by (129) is called a 'span'. ${ }^{63}$

(129) A sequence $<X_{n}, \ldots, X_{1}>$ is a span if and only iff for each pair $\left\langle X_{p}, X_{p-1}>\right.$ in the sequence, $X_{p-1}$ is (the head of) the complement of $X_{p}$.

In (128), $\langle X, Y\rangle$ is a span, but $\langle X, Z>$ isn't.

In accordance with Brody's 'Mirror Axiom', the morphemes lexicalizing the heads in a span spell out as a 'morphological word' where the linear order of these morphemes is the mirror image of the dominance relation between the corresponding heads. Thus, the span $\langle X, Y\rangle$ in (128) with $X$ and $Y$ spelled out by $A$ and $B$ will surface as the morphological word $B A$.

Hall also allows a span to be lexicalized by single morpheme (see footnote 28). For example, the span $\langle X, Y\rangle$ in (128) might also be spelled out as $C$, if the lexicon contains a morpheme with the entry $C$ $\leftrightarrow\langle X, Y\rangle$. This assumption is a crucial element in his account of a striking contrast between Mandarin Chinese (MC) and Cantonese: In MC only bare nouns can be definite, while only nouns with a classifier are ever definite in Cantonese.

A classifier-less definite noun is assigned the structure in (130) (Hall's (261) without an AP), where D encodes definiteness:

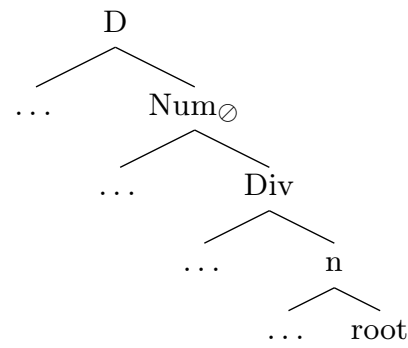

The root and all the heads merged on top of it in (130) are a span, and Hall proposes that this span is always lexicalized by a single vocabulary item in MC. That is, there is a morpheme $M_{x} \leftrightarrow<D, N u m$, Div, $\mathbf{n}$, root $_{x}>$ for each root in the language. Therefore, bare nouns can be definite in MC. (When they are indefinite, the extended projection of the nominal root doesn't contain $D$.)

\footnotetext{
63 This definition is based on Svenonius (2012b).
} 
Cantonese, on the other hand, has no morphemes capable of lexicalizing the whole span of heads in (130). Nor is it possible to lexicalize $D$ as part of a proper subspan of (130) due to a general condition we return to below. If $D$ must be spelled out as part of a larger morphological word, as Hall (2015: 162) claims ${ }^{64}$ it follows that Cantonese cannot have bare definite nouns.

On the other hand, a noun combining with a classifier can be definite in Cantonese suggesting that Cantonese allows $D$ to be lexicalized by the classifier. Since lexicalization in this theory targets spans rather than constituents, this might be achieved by morphemes lexicalizing just the span $<D, N u m_{\oslash}$, Div $>$ in (130) leaving $<n$, root $>$ to be lexicalized separately. Cantonese would then differ from MC by having morphemes $\mathrm{M}_{x} \leftrightarrow<D, N u m_{\oslash}$, Div $v_{x}>$ corresponding to different classifier $X$ merged in Div.

Hall, however, rejects this analysis, and adopts the view that lexicalization cannot break up a single projection line into two (or more) spans (his 'No Chop' condition) as would be the case if lexicalization proceeded as in (131). ${ }^{65}$

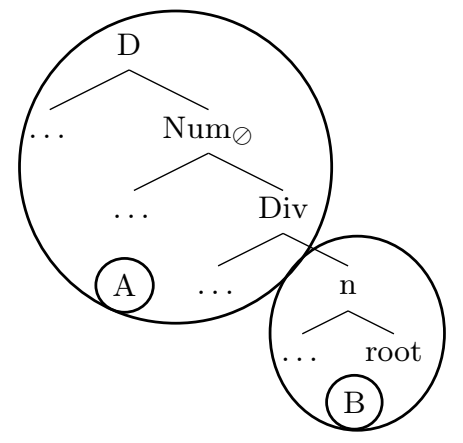

Hence, at this point his analysis converges with an analysis like ours that only allows lexicalization to apply to constituents. He concludes that a classifier must correspond to a separate extended projection standing in a Spec/head relation to the projection of the host noun in a way recalling our proposal for the Bantu class prefixes.

More specifically, he proposes (132) as the structure underlying definite classifier-noun sequences in Cantonese: ${ }^{66}$

64 Suppose, however, that the general condition alluded to above allows $D$ to be lexicalized all by itself in (130), while some other morpheme $B$ lexicalizes the lower heads. In that case, the morpheme $A$ lexicalizing $D$ would come together with $B$ in a morphological word $B A$, and $D$ would consequently be spelled out as part of a morphological word. So Cantonese must not have any morpheme capable of lexicalizing $D$ in isolation from the rest of the span. 65 The 'No Chop' condition must allow 'Chops' where each head on the projection line is lexicalized by a separate morpheme, since otherwise Brody's mirrored morphological words could never arise. Hall's analysis of classifier languages where $D$ is spelled out as a suffix on the classifier seems to rely on Chops being possible also when only the highest head is spelled out by a separate morpheme while a single morpheme lexicalizes all the lower heads on the projection line. These two exceptions to the 'No Chop' condition follow automatically from deriving 'No Chop' by restricting lexicalization to constituents.

${ }^{66}$ It is not clear to us why Hall posits two segments of Div here. The inclusion of the head uDiv in (132) is based on Adger's (2015) idea that a specifier must be identified as such by having an uninterpretable feature matching an interpretable feature on the mother node. 


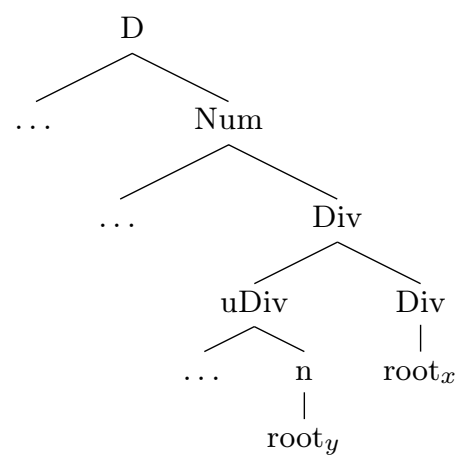

The classifier is root $_{x}$, the lexical item needed to initiate the second projection of nominal functional structure. Here, D, Num, Div and root $_{x}$ form a span $\left\langle D, N u m\right.$, Div, root $_{x}>$ containing all the heads in the extended projection of $\operatorname{rot}_{x}$. Hence, they can be lexicalized by a single morpheme $M \leftrightarrow<D, N u m$, Div, $\operatorname{root}_{x}>$ without lexicalization breaking up a single projection line. Assuming that Cantonese has such morphemes, (132) will give rise to the definite classifier-noun sequences in this language ${ }^{67}$ If $\mathrm{MC}$ has no such morphemes as opposed to morphemes $M_{x} \leftrightarrow<D, N u m_{\oslash}$, Div, n, $\left.\operatorname{root}_{x}\right\rangle, D$ cannot be lexicalized in (132) in MC, since no span includes both $D$ and [ $n$ [ $\left.\left.\operatorname{root}_{x}\right]\right]$ in (132) ${ }^{68}$ It follows that only bare nouns arising from (130) can be definite in MC.

In (132), the projection of the host noun is a specifier on the projection line of the classifier, i.e. [ Div [ root $\left._{x}\right]$ ], whereas we have taken the Bantu class prefixes to be specifiers on the projection line of the host noun. As near as we can tell, however, (132) can perfectly well be replaced with (133) even in the context of Hall's theoretical assumptions. ${ }^{69}$

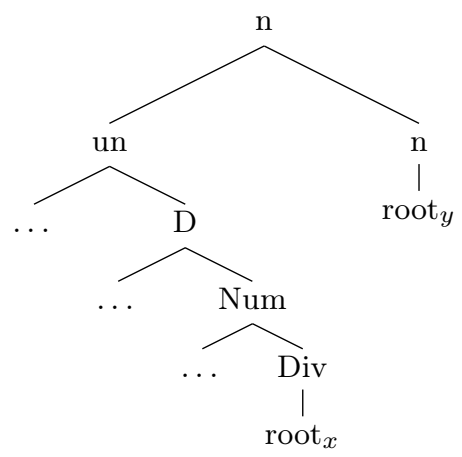

In (133), all the heads in the specifier rooted in $\operatorname{root}_{x}$ can be spelled out by a single morpheme even if lexicalization is restricted to constituents, as we assume.

\footnotetext{
67 A morpheme $M \leftrightarrow<D, N u m$, Div, root $_{x}>$ could not also lexicalize the entire span in (130) giving rise to definite bare nouns in Cantonese. The structure in (130) contains $n$, but $\left\langle D, N u m\right.$, Div, root $\left._{x}\right\rangle$ does not, and a morpheme can only lexicalize subspans of the span its lexical entry associates it with. This is the spanning counterpart of the Subset Principle introduced in section 3.4.

68 Notice that using $M_{x} \leftrightarrow<D, N u m$, Div, $n$, root $_{x}>$ to lexicalize just the subspan $<$ D, Num, Div $>$ in $(132)$ to the exclusion of root $_{x}$ would have the effect of breaking up a single projection line.

69 Hall notes that bare nouns are neutral with respect to the singular/plural opposition in both MC and Cantonese, while nouns combining with classifiers are always singular in MC and can be either singular or plural (with the classifier $d i$ ) in Cantonese. This is consistent with placing a specified Num only in the projection of the Div - root, as in (133)

To obtain the correct linearization classifier > noun from (132) in Hall's system, a spell-out diacritic @ must be placed on Num or D. If linearization is based on (133), the correct morpheme order follows directly from the basic linearization principles assumed by Hall.
} 
Leaving aside differences at a more detailed level of analysis, a non-telescoped representation of (133) would look quite similar to the structures we have proposed for prefix - noun combinations in Bantu. ${ }^{70}$

In (133), the structure underlying a (definite) classifier is merged as a specifier on the projection line of the host noun, and this specifier is the extended projection of a lexical head. As represented in (133) (and (132)), this lexical head is a root immediately dominated by Div, recalling Kihm's (2001) idea the class prefixes in Manjaku correspond to $n$ containing a lexical root, a protonoun in his terminology. In our analysis of the Bantu prefixes, this lexical head, the protonoun, is represented as an $N$.

Like us, Hall is led by purely theoretical assumptions to posit a lexical head inside the Chinese classifiers, but he then also shows that a number of empirical facts support this analytical decision. In particular, he observes that there is some flexibility as to which nouns combine with which classifiers, but when a noun co-occurs with a classifier other than its 'primary classifier', certain semantic effects are detected in a way comparable to what happens when a Bantu noun combines with a 'secondary prefix'. This, he argues, is best understood if the different classifiers contain different lexical roots whose semantics composes with the semantics of the host nouns, although this may be overridden by idiomatic interpretations. This is essentially the line of argument followed here for the Bantu class prefixes (see section 5) and by Kihm for class prefixes in Manjaku. We find it significant that similar analyses of different languages should emerge in this way even in the context of different theoretical assumptions.

\subsection{Summary}

In this section, we began by explaining why our the theoretical assumptions adopted in our account of the nominal prefixes in Bantu predict that all nominal portmanteau prefixes must be taken to lexicalize specifiers where the number head and the gender head are projected on top of an $N$ different from the noun hosting the prefix. Then, we argued that the 'protonoun' contained in $n$ in Kihm's (2001) analysis of Manjaku should be identified with this $N$. If so, the Manjaku facts motivating Kihm's analysis can be taken to bear out our prediction.

We have also shown that our theoretical assumptions predict an asymmetry between portmanteau prefixes and portmanteau suffixes: Portmanteau suffixes may also be specifiers containing a protonoun, but also allow an analysis without a protonoun. The contrast between the Somali suffixes, as characterized by Lecarme (2002) and the Romance gender/number-marking suffixes, as characterized by Kihm (2001), would appear to verify this prediction.

Finally, we showed that Hall's (2015) conclusions about Chinese classifiers also seem to converge with ours in important respects. We would like to emphasize that the prediction that seems to be borne out in the languages discussed in this section as well as in the Southern Bantu languages examined in the previous sections ultimately stems from the claim adopted in section 3.3 that a non-trivial set of terminals can be lexicalized by a single morpheme only if the relevant terminals form a constituent. A 'spanning' approach would not have led to the same prediction.

${ }^{70}$ Hall adopts Adger's (2015) proposal that a specifier cannot have a label higher in the functional sequence than the phrase it is a specifier of. As it stands, (133) violates this constraint insofar as D, Num and Div are higher than $n$ in the functional sequence. A representation conforming to the constraint would be (i) with $D$ silent on the projection line from $\operatorname{root}_{y}$ :

(i)

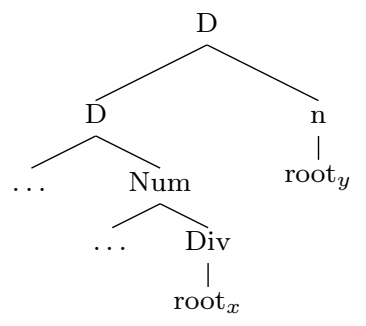

This would also be perfectly in line with our representations for prefixed nouns in Bantu. 
Needless to say, it remains to be seen whether cross-linguistic research will bear out our predictions more generally.

\section{Conclusions}

The basic empirical claim we make is that noun class prefixes in the Southern Bantu languages discussed here, are morphemes lexicalizing a syntactic phrase merged on the projection line of the noun. This syntactic phrase is the extended projection of a second noun acting as a classifier. This conclusion was initially limited to certain plural prefixes and rested on two premises. Some plural prefixes cannot be taken to inherit the gender of the (overt) noun they combine with. But gender-features must always originate from a noun.

The general theory of lexicalization that we used to account for the fact that the classifying noun is always silent in the relevant plural prefixes, ultimately led us to claim that all class prefixes lexicalize specifiers bottoming out in a noun. Then, we argued that this conclusion also provides an optimal way of dealing with the relationship between primary and secondary prefixes in Bantu.

As a corollary of our conclusion, the pairing of nouns and class prefixes cannot be captured by positing transfer of gender features from the noun to the prefix. In fact, no reason remains to think that nouns have gender at all in Bantu. Therefore, we fall back on the more traditional view that prefixes (viewed as classifiers) are paired with nouns on the basis of semantic compatibility or by being listed as phrasal idioms in the lexicon.

Acknowledgements The field work carried out by Knut Tarald Taraldsen and Lucie Taraldsen Medová was supported with a grant from Troms $\varnothing$ Forskningsstiftelse to the project The morphosyntax of Bantu nouns (project number A3792). We want to thank the native speakers of Xhosa and Zulu who shared their intuitions with us, with a special thank to Khanyiso Mfuleni. We are also indebted to Surena Du Plessis and Marianna Visser, who helped us set up the sessions with our consultants in Stellenbosch, and to Claire Halpert and Jochen Zeller for assisting us in finding consultants in Durban. Colleagues in Troms $\varnothing$ have provided useful comments on various issues discussed in this article, and we are also grateful to anonymous reviewers whose comments have forced us to sharpen the analyses and to make the exposition more precise. Special thanks go to Michal Starke for many discussions of the theoretical issues involved. The usual disclaimers apply.

\section{References}

Abels, Klaus, and Peter Muriungi. 2008. The focus particle in Kîitharaka: Syntax and semantics. Lingua 118: 687-731.

Acquaviva, Paolo. 2008. Lexical Plurals: A Morphosemantic Approach: A Morphosemantic Approach. Oxford University Press.

Adger, David. 2013. A syntax of substance. Vol. 64 of Linguistic Inquiry Monograph. MIT Press.

Bachetti, Cláudio. 2006. Gramática da língua Ronga. Maputo: Paulinas Editorial.

Bresnan, Joan, and Sam A Mchombo. 1995. The lexical integrity principle: Evidence from Bantu. Natural Language ES Linguistic Theory 13 (2): 181-254.

Brody, Michael. 1997. Mirror theory. Ms., University College London.

Brody, Michael. 2000. Mirror theory: Syntactic representation in perfect syntax. Linguistic Inquiry 31 (1): 29-56.

Buell, Leston. 2009. The distribution of the Nguni augment: A review. Ms.,. http://www.fizzylogic.com/users/bulbul/school/buell-nguni-augment.pdf.

Caha, Pavel. 2009. The nanosyntax of case. PhD diss, CASTL Troms $\varnothing$. Available at http://ling.auf.net/lingBuzz/000956.

Caha, Pavel, and Marina Pantcheva. 2014. Locatives in Shona and Luganda. Available at http://ling.auf.net/lingbuzz/002220.

Carstens, Vicki. 1991. The morphology and syntax of determiner phrases in Kiswahili. PhD diss, University of California, Los Angeles 1991.

Carstens, Vicki. 1997. Null nouns in Bantu locatives. The Linguistic Review 14 (4): 361-410.

Carstens, Vicki. 2008. DP in Bantu and Romance. In The Bantu-Romance Connection, 131-166. Amsterdam: John Benjamins.

Carstens, Vicki. 2011. Hyperactivity and hyperagreement in Bantu. Lingua 121 (5): 721-741. 
Chomsky, Noam. 2013. Problems of projection. Lingua 130: 33-49.

Chung, Inkie. 2009. Suppletive verbal morphology in Korean and the mechanism of vocabulary insertion. Journal of Linguistics 45 (3): 533-567.

Cinque, Guglielmo. 2005. Deriving Greenberg's universal 20 and its exceptions. Linguistic Inquiry 36 (3): 315-332.

Corbett, Greville G, and Alfred D. Mtenje. 1987. Gender agreement in Chichewa. Studies in African Linguistics 18 (1): $1-38$.

Crisma, Paola, Lutz Marten, and Rint Sybesma. 2011. The point of Bantu, Chinese and Romance nominal classification. Italian Journal of Linguistics 23 (2): 251-299.

De Vos, Mark, and Hazel Mitchley. 2012. Subject marking and preverbal coordination in Sesotho: A perspective from Optimality Theory. Southern African Linguistics and Applied Language Studies 30 (2): 155-170.

Déchaine, Rose-Marie, Raphaël Girard, Calisto Mudzingwa, and Martina Wiltschko. 2014. The internal syntax of Shona class prefixes. Language Sciences 43: 18-46.

Diercks, Michael, Lindsey Meyer, and Mary Paster. 2015. Agreement with conjoint arguments in Kuria. Studies in African Linguistics 44 (1): 27-56.

Fortune, George. 1955. An analytical grammar of Shona. Longmans, Green.

Georgi, Doreen, and Gereon Müller. 2010. Noun-Phrase Structure by Reprojection. Syntax 13 (1): 1-36.

Givón, Talmy. 1970. The resolution of gender conflicts in Bantu conjunction: when syntax and semantics clash, 250-261. Papers from the sixth regional meeting, Chicago Linguistic Society.

Hall, David. 2015. Spelling out the noun phrase: Interpretation, word order, and the problem of 'meaningless movement'. PhD diss, Doctoral dissertation, Queen Mary University of London.

Halpert, Claire. 2015. Argument licensing and agreement. Oxford University Press.

Katamba, Francis. 2003. Bantu nominal morphology. In The bantu languages, 103-120. Routledge London and New York.

Kayne, Richard S. 1994. The antisymmetry of syntax. Vol. 25 of Lingustic Inquiry Monograph. MIT Press.

Kayne, Richard S. 2005. A note on the syntax of quantity in English. In Movement and silence, 176-214. New York: Oxford University Press.

Kayne, Richard S. 2016. What is suppletive allomorphy? On went and *goed in English. Ms., NYU.

Kihm, Alain. 2005. Noun class, gender, and the lexicon-syntax-morphology interfaces: A comparative study of Niger-Congo and Romance languages. The Oxford handbook of comparative syntax 459: 512.

Lecarme, Jacqueline. 2002. Gender 'polarity': Theoretical aspects of Somali nominal morphology. In Many morphologies, ed. Paul Boucher, 109-141. Somerville, MA: Cascadilla Press.

Manzini, M Rita, and Leonardo M Savoia. 2005. Morfosintassi delle varietà italiane e romance. Edizioni dell'orso. Alessandria.

Marten, Lutz. 2000. Agreement with conjoined noun phrases in Swahili. In Afrikanistische Arbeitspapiere: Swahili Forum VII, Vol. 64, 75-96.

Marten, Lutz. 2005. The dynamics of agreement and conjunction. Lingua 115 (4): 527-547.

McCawley, James D. 1968. Lexical insertion in a transformational grammar without Deep structure. In Papers from the fourth regional meeting of the Chicago Linguistic Society. University of Chicago.

McCreight, Katherine, and Catherine V Chvany. 1991. Geometric representation of paradigms in a modular theory of grammar. In Paradigms: The economy of inflection, ed. Frans Plank, 91-112. Berlin, New York: Mouton de Gruyter.

Myers, Scott P. 1987. Tone and the Structure of Words in Shona. PhD diss, University of Massachussetts, Amherst.

Pantcheva, Marina Blagoeva. 2011. Decomposing Path. The Nanosyntax of Directional Expressions. PhD diss, CASTL Troms $\varnothing$.

Roberts, Ian G. 2010. Agreement and head movement: Clitics, incorporation, and defective goals. Vol. 59 of Lingustic Inquiry Monograph. MIT Press.

Schadeberg, Thilo C. 2001. Number in Swahili grammar. In Afrikanistische Arbeitspapiere: Swahili Forum VIII, Vol. 66, 7-16.

Schwarzschild, Roger. 1996. Pluralities. Dordrecht: Kluwer Academic Publishers.

Sportiche, Dominique. 1996. Clitic constructions. In Phrase structure and the lexicon, eds. Johan Rooryck and Laurie Zaring, 213-276. Springer.

Sportiche, Dominique. 2005. Division of labor between Merge and Move: Strict locality of selection and apparent reconstruction paradoxes. Available at http://ling.auf.net/lingbuzz/000163.

Starke, Michal. 2004. On the inexistence of specifiers and the nature of heads. In Structures and Beyond: The Cartography of Syntactic Structures, ed. Adriana Belletti, Vol. 3, 252-268. New York: Oxford University Press.

Starke, Michal. 2009. Nanosyntax - A short primer to a new approach to language. Nordlyd. Special issue on Nanosyntax 36 (1): 1-6. Available at http://ling.auf.net/lingbuzz/001230.

Starke, Michal. 2011. Towards an elegant solution to language variation: Variation reduces to the size of lexically stored trees. Available at http://ling.auf.net/lingbuzz/001183.

Starke, Michal. to appear. Complex left branches, spell-out and prefixes. In Exploring nanosyntax, eds. L. Baunaz; K. De Clercq; L. Haegeman and E. Lander. New York: Oxford University Press.

Stump, Gregory T. 1989. A note on Breton pluralization and the Elsewhere Condition. Natural Language E 
Linguistic Theory 7 (2): 261-273.

Svenonius, Peter. 2012a. Merge, Project and Bundle. Ms., CASTL University of Troms $\varnothing$.

Svenonius, Peter. 2012b. Spanning. Ms., CASTL University of Troms $\varnothing$.

Taraldsen, Knut Tarald. 2009. Lexicalizing number and gender in Colonnata. Ms., CASTL University of Troms $\varnothing$.

Taraldsen, Knut Tarald. 2010. The nanosyntax of Nguni noun class prefixes and concords. Lingua 120 (6): 15221548.

Van der Spuy, Andrew. 1993. Dislocated noun phrases in Nguni. Lingua 90 (4): 335-355.

Voeltz, Erhard. 1971. Surface constraints and agreement resolution: Some evidence from Xhosa. Studies in African Linguistics 2 (1): 37.

Williams, Edwin. 2003. Representation theory. Current Studies in Linguistics. MIT Press. 